\title{
GPU Domain Specialization via Composable On-Package Architecture
}

\author{
YAOSHENG FU, EVGENY BOLOTIN, NILADRISH CHATTERJEE, DAVID NELLANS, and \\ STEPHEN W. KECKLER, NVIDIA, USA
}

\begin{abstract}
As GPUs scale their low-precision matrix math throughput to boost deep learning (DL) performance, they upset the balance between math throughput and memory system capabilities. We demonstrate that a converged GPU design trying to address diverging architectural requirements between FP32 (or larger)-based HPC and FP16 (or smaller)-based DL workloads results in sub-optimal configurations for either of the application domains. We argue that a Composable On-PAckage GPU (COPA-GPU) architecture to provide domain-specialized GPU products is the most practical solution to these diverging requirements. A COPAGPU leverages multi-chip-module disaggregation to support maximal design reuse, along with memory system specialization per application domain. We show how a COPA-GPU enables DL-specialized products by modular augmentation of the baseline GPU architecture with up to $4 \times$ higher off-die bandwidth, $32 \times$ larger on-package cache, and $2.3 \times$ higher DRAM bandwidth and capacity, while conveniently supporting scaleddown HPC-oriented designs. This work explores the microarchitectural design necessary to enable composable GPUs and evaluates the benefits composability can provide to HPC, DL training, and DL inference. We show that when compared to a converged GPU design, a DL-optimized COPA-GPU featuring a combination of $16 \times$ larger cache capacity and 1.6 $\times$ higher DRAM bandwidth scales per-GPU training and inference performance by $31 \%$ and $35 \%$, respectively, and reduces the number of GPU instances by $50 \%$ in scale-out training scenarios.
\end{abstract}

CCS Concepts: • Computer systems organization $\rightarrow$ Processors and memory architectures; Neural networks;

Additional Key Words and Phrases: GPU computing, multi-chip module

\section{ACM Reference format:}

Yaosheng Fu, Evgeny Bolotin, Niladrish Chatterjee, David Nellans, and Stephen W. Keckler. 2021. GPU Domain Specialization via Composable On-Package Architecture. ACM Trans. Arch. Code Optim. 19, 1, Article 4 (December 2021), 23 pages.

https://doi.org/10.1145/3484505

\section{INTRODUCTION}

Deep learning (DL) has revolutionized computer vision, natural language processing, speech recognition, and recommendation systems [7, 20, 26, 27] and reshaped the automotive, robotics, e-commerce, and healthcare industries [23, 72, 73, 75]. GPUs have become the de facto platform of choice for DL applications due to their relative ease of programming, rich set of optimized

Authors' address: Y. Fu, E. Bolotin, N. Chatterjee, D. Nellans, and S. W. Keckler, NVIDIA Corporation, 2788 San Tomas Expy, Santa Clara, CA USA 95051; emails: \{yfu, ebolotin, nchatterjee, dnellans, skeckler\}@nvidia.com.

Permission to make digital or hard copies of all or part of this work for personal or classroom use is granted without fee provided that copies are not made or distributed for profit or commercial advantage and that copies bear this notice and the full citation on the first page. Copyrights for components of this work owned by others than ACM must be honored. Abstracting with credit is permitted. To copy otherwise, or republish, to post on servers or to redistribute to lists, requires prior specific permission and/or a fee. Request permissions from permissions@acm.org.

(C) 2021 Association for Computing Machinery.

1544-3566/2021/12-ART4 \$15.00

https://doi.org/10.1145/3484505

ACM Transactions on Architecture and Code Optimization, Vol. 19, No. 1, Article 4. Publication date: December 2021. 


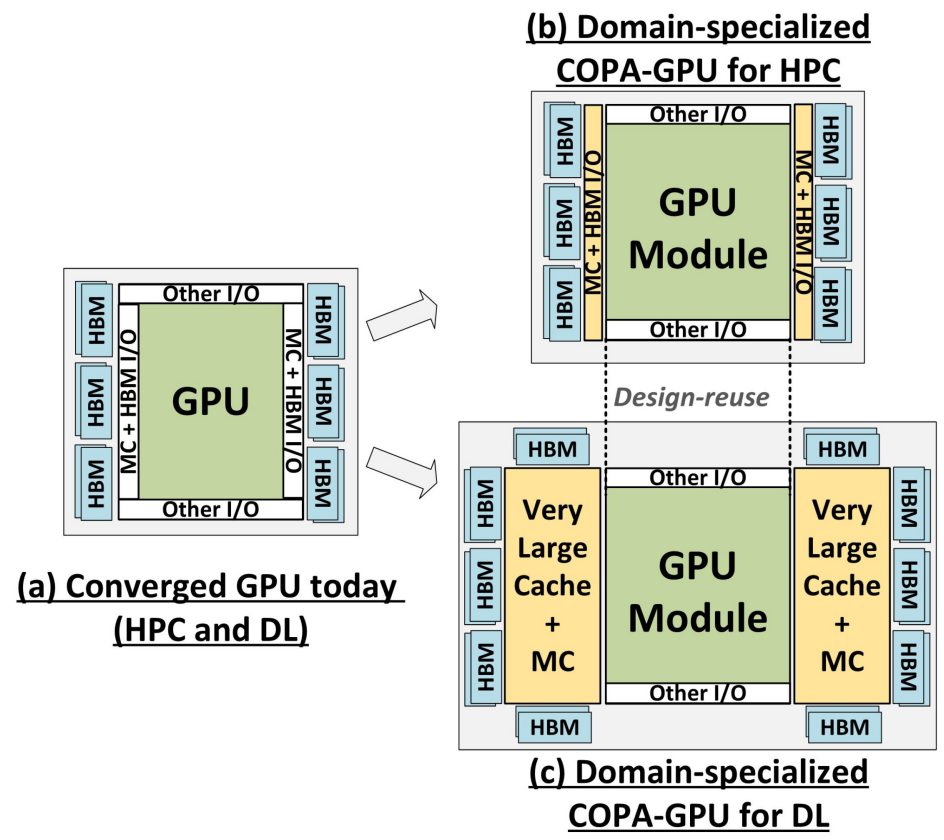

Fig. 1. (a) A converged monolithic GPU for HPC and DL. (b) An HPC-specialized COPA-GPU with similar capabilities to converged GPU. (c) A DL-specialized COPA-GPU featuring a large capacity, high-bandwidth on-package cache, and scaled DRAM resources.

software libraries, and good balance between compute and off-chip memory bandwidth [66]. GPU acceleration can reduce the training time of DL applications from months to minutes [57]. However, GPU architectures must keep evolving to satisfy the unprecedented expansion in DL computing demand, fueled by the growing complexity of both model and dataset sizes [40].

GPU vendors have long enjoyed the benefits of shrinking process technologies that increased transistor density, while also manufacturing ever larger dies [58,61, 63, 64, 66]. Thanks to technology scaling, architects have had ample resources to add domain-specialized hardware to computer-graphics-oriented GPUs, resulting in today's converged GPU design that serves multiple application domains. To cater to High-Performance Computing (HPC), GPUs added costly high-precision arithmetic (FP64) units, advanced error detection and correction hardware, highbandwidth memories (HBMs), and fast I/O such as NVIDIA's NVLINK [6, 62, 64, 66]. Similarly, specialized hardware such as NVIDIA Tensor Cores and AMD Matrix Cores were added for DL, while RT Cores were added to accelerate next-generation ray tracing capabilities [6, 64-66].

The recent dramatic improvement in low-precision math throughput on GPUs is driven by research work showing the feasibility of high-accuracy DL training and inference while utilizing lower-precision arithmetic [30, 44, 54]. For example, NVIDIA's V100 GPU introduced DL Tensor Cores improving FP16 throughput by $6 \times$ in one GPU generation [64]. NVIDIA's Turing GPU family went even further, integrating INT4/8 support into the Tensor Cores [65]. Most recently, NVIDIA's A100 GPU increased the FP16 throughput by an additional 2.5× over V100 [66]

This work demonstrates that converged GPU designs fail to satisfy the diverging requirements, in terms of memory bandwidth to compute throughput, between FP32/FP64-based HPC applications and low-precision-based DL networks. Moreover, the slowdown in transistor scaling along with reticle limitations in silicon lithography is restricting transistor and die area growth, making it ever harder to deliver competitive performance in multiple application domains with a single 
converged GPU design. Future off-chip bandwidth scaling is also at risk as the die edge scales sub-linearly with increasing die size, limiting the off-chip DRAM and I/O bandwidth available to individual dies [8].

The growing importance of DL domain-specific acceleration in the datacenter is demonstrated by a proliferation of accelerators targeted exclusively at DL algorithms [25, 34, 36, 38, 43, 46, 47]. We believe that the combination of technology trends and an ultra-competitive DL landscape has created an inflection point at which GPU manufacturers must embrace more domain-specialized GPU designs to continue providing competitive performance, while also maximizing design reuse and minimizing non-recurring engineering (NRE) costs.

In this work we examine historical trends and project future ratios that show a strong divergence between high- and low-precision math throughput on GPUs. We show that this gap makes it difficult to design a memory system that efficiently supports both HPC and DL use cases. Thus, we propose a Composable On-PAckage GPU (COPA-GPU) architecture as a practical solution for building a new class of domain-optimized GPUs. Leveraging multi-chip module (MCM) integration $[8,12,28,39,55,59]$ along with emerging circuit technology innovations $[28,48,52]$, we propose to replace the single converged GPU that serves both HPC and DL domains with composable, semi-specialized designs as shown in Figures 1(b) and 1(c).

A COPA-GPU that is specialized for HPC (similar to Figure 1(b)) uses the baseline GPU module (GPM) and a memory system module providing similar or scaled-down capabilities as in the converged GPU today. Augmenting the memory system modules with a very large on-package cache accessible at high on-package bandwidth and additional off-chip DRAM resources (Figure 1(c)) results in a substantially different COPA-GPU design point that is well suited for bandwidth-hungry DL workloads. This level of specialization is not free, as it requires both intelligent architectural disaggregation of the GPU memory system and employing emerging circuit and packaging techniques. This work aims to shed light on the architectural choices available to designers when building and specializing composable GPU designs.

This work makes the following contributions:

- We examine historical GPU trends and perform a detailed performance analysis of diverging HPC and DL benchmarks. We show that due to the converged nature of GPUs and future DL scaling requirements, the GPU's memory bandwidth will become the primary performance bottleneck for GPU-based DL training and inference, while being under-utilized for most HPC applications.

- We propose the development of domain-specialized composable GPU architectures. COPAGPUs provide high levels of GPU design reuse across the HPC and DL domains, while enabling specifically optimized products for each domain. We describe both the architectural modifications and the landscape of technologies needed to enable COPA-GPUs.

- We evaluate the performance potential of COPA-GPU in the context of DL training and inference and show that very large cache capacity can dramatically improve DL-inference, but both cache and DRAM improvements (available only through COPA designs) are necessary to significantly improve DL-training.

- Finally, we propose a specific COPA-GPU design boosting per-GPU training and inference performance by up to $31 \%$ and $35 \%$, respectively, while also significantly reducing the cost of scale-out GPU training in datacenters.

\section{MOTIVATION AND BACKGROUND}

The commercial importance of deep learning is undeniable, and numerous companies are now designing targeted DL training and inference accelerators, such as Google's TPU [36-38], 
Table 1. GPU and DL Accelerator Compute and Memory System Trends, Including a Forward-looking GPU Projection (GPU-N)

\begin{tabular}{|l|c|c|c|c|c|}
\hline $\begin{array}{c}\text { GPU } \\
\text { Architecture }\end{array}$ & $\begin{array}{c}\text { FP32 } \\
\text { [TFLOPS] }]\end{array}$ & $\begin{array}{c}\text { FP16 } \\
\text { [TFLOPS] }\end{array}$ & $\begin{array}{c}\text { L2 } \\
{[\mathrm{MB}]}\end{array}$ & - & $\begin{array}{c}\text { DRAM BW } \\
{[\mathrm{GB} / \mathrm{s}]}\end{array}$ \\
\hline NVIDIA P100 & 11 & 21 & 4 & - & 732 \\
\hline NVIDIA V100 & 16 & 125 & 6 & & 900 \\
\hline NVIDIA A100 & 20 & 312 & 40 & - & 1,555 \\
\hline GPU-N & 24 & 779 & 60 & - & 2,687 \\
\hline \hline & FP32 & FP16 & $\begin{array}{c}\text { On-chip } \\
\text { Memory } \\
\text { Accelerator }\end{array}$ & $\begin{array}{c}\text { On-chip } \\
\text { Memory BW } \\
{[\text { TFLOPS] }}\end{array}$ & $\begin{array}{c}\text { DRAM BW } \\
{[\text { TFLOPS] }}\end{array}$ \\
\hline Graphcore IPU & 31 & 125 & 304 & 45 & N/A $]$ \\
\hline Graphcore IPUv2 & 62 & 250 & 896 & 45 & N/A \\
\hline Cerebras WSE & - & - & 214 & 107 & N/A \\
\hline Cerebras WSE-2 & - & - & 476 & 238 & N/A \\
\hline Groq TSP & - & 250 & 220 & 80 & N/A \\
\hline Google TPUv2 & 5 & 46 & 32 & - & 700 \\
\hline Google TPUv3 & 14 & 123 & 32 & - & 900 \\
\hline Google TPUv4i & - & 138 & 144 & - & 614 \\
\hline
\end{tabular}

We report the attributes of Cerebras as 1/84 tiles in a wafer to provide area-similar comparisons.

Graphcore's IPU [34, 43], Cerebras' WSE [46, 47], and Groq's TSP [25]. These application-specific architectures need not maintain a legacy of high performance in other domains, allowing them to make heavily specialized architectural choices compared to GPU architectures that currently serve multiple domains. To better understand the divergence of GPU and DL accelerator design trends, we examined recent GPU scaling trends, projected a plausible future GPU configuration, and now compare it to several dedicated DL accelerators.

\subsection{Architecture Trends and Projections}

Table 1 summarizes several key compute and memory system characteristics across the three most recent generations of NVIDIA Tesla GPUs targeting both HPC and DL domains. We then forwardproject the hardware capabilities of a hypothetical next-GPU configuration (GPU-N) using evolutionary scaling. We calculate the compute and memory bandwidth of GPU-N by linearly extrapolating these parameters from V100 to A100 $[64,66]$. We calculate the GPU-N L2 capacity using the lower scaling rate of $1.5 \times$ from P100 [63] to V100 (instead of $6.7 \times$ from V100 to A100) since GPU-N is highly unlikely to be able to fit hundreds of MB of SRAM on a single GPU die. Note that we are not trying to accurately project the exact configuration of a future GPU, but rather demonstrate the effect of expected scaling rates on compute and memory bandwidths. Table 1 makes it clear that while the memory bandwidth to FP32 throughput ratios have generally increased across GPU generations (from $67 \times$ in P100 to $112 \times$ in GPU-N), the memory bandwidth to FP16 ratio has diminished to only $3.4 \times$ in GPU-N.

In addition to several GPU generations, Table 1 provides attributes of several DL accelerators. The Graphcore, Cerebras, and Groq products all feature very large on-chip SRAMs as main memory, while Google TPUs use off-chip DRAM (HBM). In contrast to GPUs and TPUs, DL accelerators with DRAM-less memory system can provide substantially higher memory bandwidth to FP16 ratios reaching $180 \times$ and $320 \times$ in IPUv2 and TSP, respectively, albeit providing much lower total memory capacity that may prove to be inadequate for rapidly growing memory footprint demands 


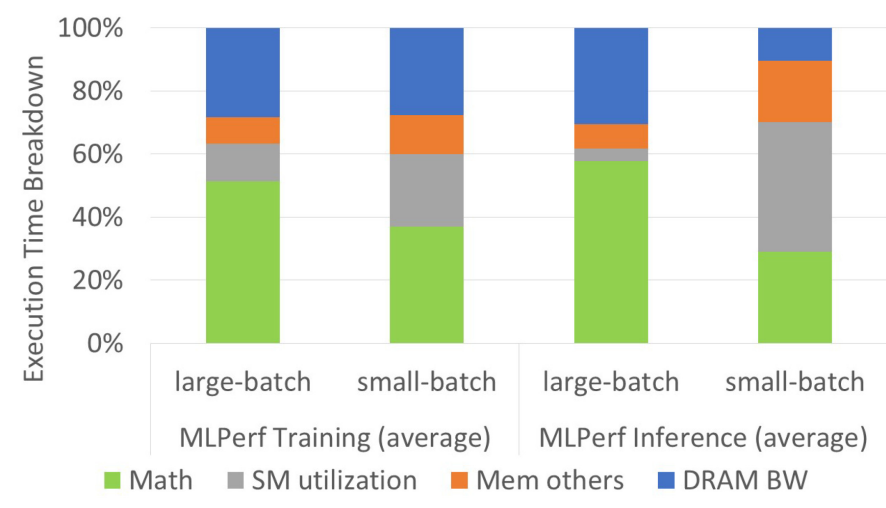

Fig. 2. GPU-N performance bottleneck analysis using the MLPerf DL training and inference suites, for both large-batch and small-batch settings.

of DL applications [40]. The designers of the future DL training and inference platforms will have to strike the right architectural balance between relatively large on-package SRAM caches for performance and energy efficiency and high-capacity DRAM to provide an adequate amount of device memory suitable for the growing DL requirements.

\subsection{Diverging DRAM Bandwidth Requirements}

To understand the diverging DRAM bandwidth requirements of future GPUs, Figure 2 presents a simulation performance bottleneck analysis of the DL workloads from the MLPerf suite (details described later in Section 4.1) for both small and large batch sizes on GPU-N. Figure 2 presents a breakdown of the total execution time, with each colored bar segment representing the performance overhead introduced by a hardware component (or a group of related hardware components) during execution. For example, the blue "DRAM BW" bars show the performance overhead attributed to non-ideal DRAM bandwidth when compared to infinite DRAM bandwidth. Similarly, the orange bars represent the performance penalty caused by all other components in the memory subsystem being non-ideal in terms of bandwidth and latency. The gray bars represent the performance penalty caused by dynamic SM under-utilization (idle SMs) versus an ideal GPU with $100 \%$ SM utilization, reflecting inefficiencies such as imperfect work scheduling or lack of sufficient parallelism (sometimes only in phases) within the workload itself. Finally, the green bars show the relative execution time spent in Math units, which should be $100 \%$ utilized in the ideal case.

Our bottleneck analysis is conducted by gradually idealizing one or more architectural features of the system starting with DRAM bandwidth and moving inward toward the SM, then using the performance differential between the idealized and the real system to determine the extent to which each component impacts overall performance. We start with the baseline GPU-N and first idealize the DRAM bandwidth to estimate the overhead of insufficient off-chip bandwidth. Then with the DRAM bandwidth remaining idealized, we idealize the rest of the memory subsystem, effectively modeling a perfect GPU memory system in which all memory requests return immediately to the SM. Finally, to quantify the effect of SM under-utilization due to load imbalance or an insufficient amount of work, we simulate a GPU-N with just one SM and a perfect memory system, and then divide the execution time by the original number of SMs to mimic the case where all SMs achieve full occupancy. The primary reason for gradual simultaneous idealization, as opposed to idealizing only one component at a time, is to understand the full potential performance of removing a bottleneck once all the bottlenecks that are downstream from it (until the DRAM) 


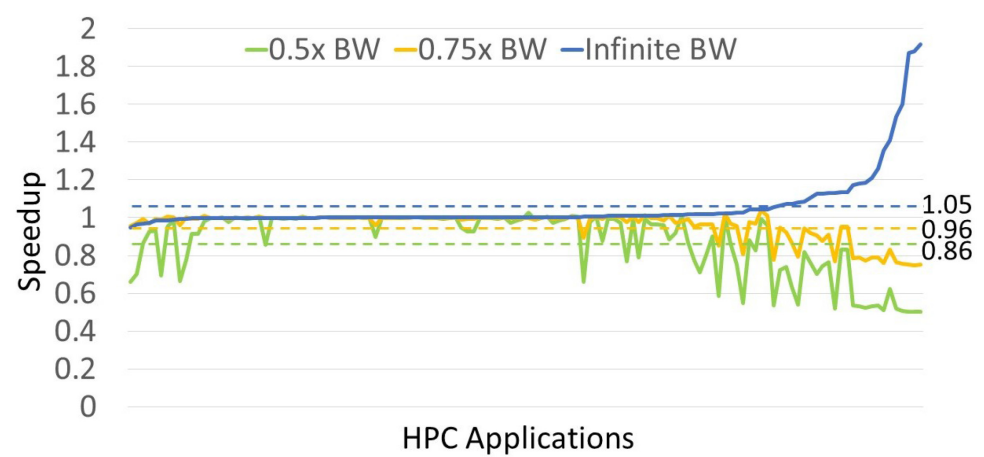

Fig. 3. Performance speedup of various DRAM bandwidths for HPC applications on a GPU-N configuration where dashed lines represent geomean speedups for corresponding configurations.

are removed. Obviously, these results can depend on the order in which the idealization steps are applied. However, as our primary focus is on the memory system, we idealize these components first to minimize the interference by idealizing other parts of the system. Our method has some overlap with the bottleneck breakdown in GSI [5]; however, our analysis goes beyond the memory subsystem and extends to SM utilization and Math units.

Figure 2 shows that indeed DRAM bandwidth is the primary performance limiter (excluding Math) for DL training on GPU-N, contributing $28 \%$ of the total execution time on average across large-batch and small-batch cases. DRAM bandwidth is also the main performance bottleneck for large-batch DL inference and accounts for $30 \%$ of the total execution time. However, at small batch sizes, SM under-utilization accounts for $41 \%$ of total execution time and serves as the primary performance bottleneck rather than DRAM bandwidth. This is because MLPerf small-batch inference does not expose enough parallelism to fill an entire GPU that was designed for the datacenter. Moreover, due to small-batch inference's relatively small memory footprints, the majority of each workload's data can be buffered on-chip, and thus DRAM bandwidth is not the primary bottleneck.

We also use the same simulator to simulate the converged GPU-N configuration across varying DRAM bandwidth settings using $130 \mathrm{HPC}$ benchmarks from differing sources including the CORAL [50] and CORAL-2 [51] benchmarks, Amber18 benchmarks [4], FUN3D [60], SPECFEM3D Cartesian [3], GROMACS [1], Laghos [49], and RELION [2]. As shown in Figure 3, in stark contrast to DL applications, most HPC applications are quite insensitive to changes in DRAM bandwidth. When DRAM bandwidth is increased to infinite, a geometric mean speedup of only $5 \%$ is achieved. When DRAM bandwidth is decreased, $0.75 \times$ BW and $0.5 \times$ BW result in $4 \%$ and $14 \%$ slowdown, respectively. This implies that future increases in DRAM bandwidth will go largely underutilized by most HPC applications if converged GPU designs targeting both DL and HPC domains remain the de facto standard.

\subsection{Very Large Caches for DL-optimized GPUs}

Because the memory bandwidth demands of DL applications are likely to exceed what evolutionary DRAM scaling can provide, GPU designers must pursue alternative methods to meet aggressive bandwidth targets. Historically, the GPU's LLC has remained relatively small because HPC workloads have good spatial locality and the majority of the off-chip bandwidth filtering potential can be captured within last-level caches that are measured in tens of megabytes. In DL workloads data locality spans multiple temporal and spatial scales, requiring much larger capacities. 


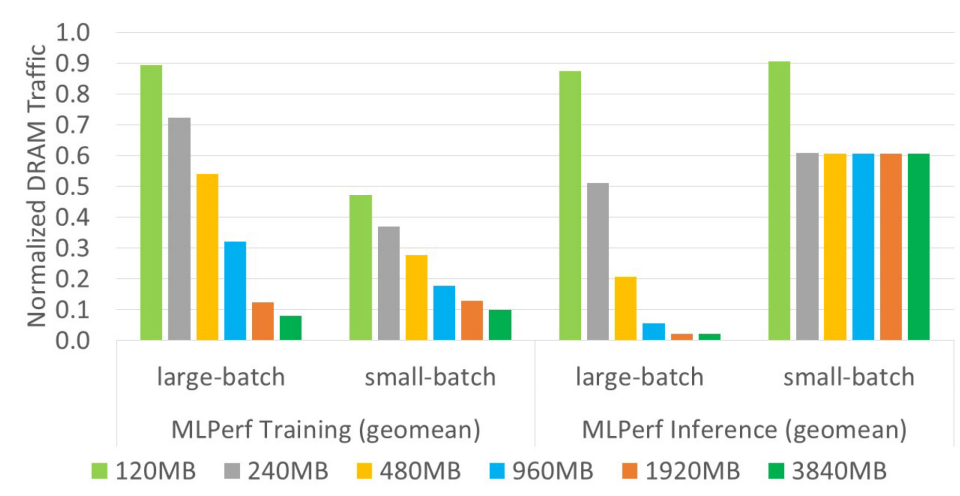

Fig. 4. DRAM traffic reduction versus LLC capacity, normalized to the baseline GPU-N configuration with $60 \mathrm{MB}$ of LLC.

To understand how much LLC capacity GPU-N would need to effectively shield the GPU's DRAM system, we examine the off-chip DRAM traffic reduction achieved when sweeping GPUN's LLC capacity from $60 \mathrm{MB}$ to $4 \mathrm{~GB}$. Figure 4 shows that doubling the LLC capacity to $120 \mathrm{MB}$ provides up to $53 \%$ reduction in off-chip DRAM traffic in DL training. Further growth to $960 \mathrm{MB}$ reduces off-chip BW demand by $82 \%$ ( $5 \times$ reduction). We will later show that DRAM traffic reduction from larger caches correlates well with improved training performance. Compared to DL training, large LLC capacities are even more beneficial for DL inference because they enable a larger portion of the workload's weights and activations to be cached on-chip. For example, in large-batch inference, a 960MB LLC achieves a 16× DRAM traffic reduction, while a 240MB LLC is sufficient to capture all available data reuse data on-chip for small-batch inference because of its smaller memory footprint.

Because modern GPUs are already area limited [8], implementing hundreds of additional megabytes of on-die LLC without severely sacrificing other functionalities is not feasible. Even if it could be done, such an architecture would be out of balance for optimal HPC performance. Because of the different sensitivities to off-chip GPU bandwidth and on-GPU cache capacities between HPC and DL workloads, we now explore the potential benefits of a composable GPU architecture that can satisfy the unique demands of each domain while maximizing design reuse through a modular on-package design.

\section{COMPOSABLE GPU ARCHITECTURE}

A COPA-GPU architecture has two primary goals: (1) to largely preserve the existing GPU architecture to minimize design effort and maximize reuse, and (2) to provide flexibility in specializing the GPU memory system for diverging requirements across application domains. Specifically, we aim to improve the GPU's memory system with substantially more cache and memory bandwidth than is required for domains such as HPC, to unlock deep learning performance on GPUs. To achieve this range of GPU system capabilities, we leverage on-package multi-chip-module integration to couple a GPU-core die with different memory system dies, each having different allocations of on-package cache and memory resources.

\subsection{Practical GPU Disaggregation with COPA-GPU}

We propose to disaggregate today's monolithic GPU into a basic GPM on-package that is reused without modifications across domain-specialized COPA-GPU instances and a domain-specialized Memory System Module (MSM) tailored for the specifics of each application domain. The main 


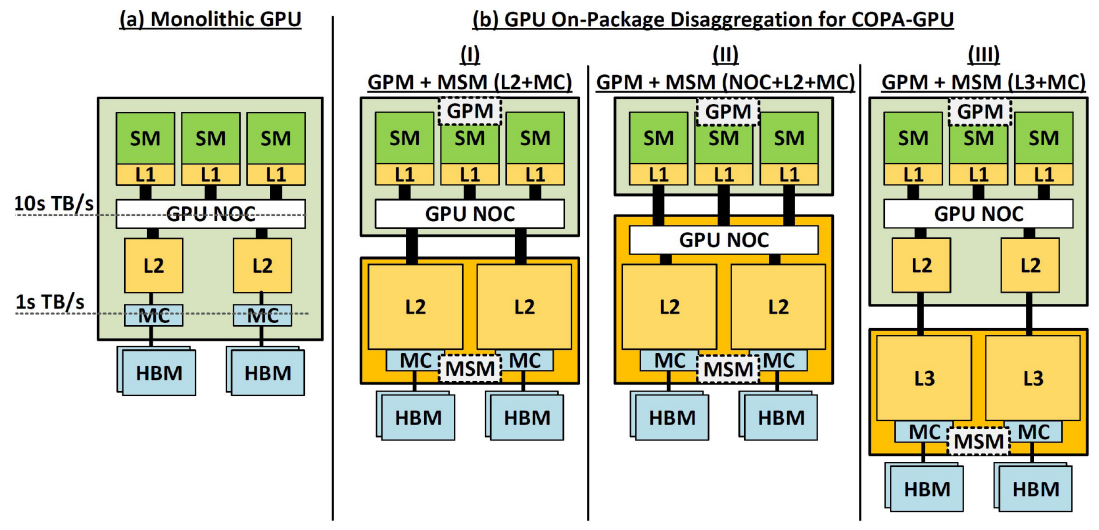

Fig. 5. GPU MCM disaggregation options for practical and composable domain specialization.

challenge with on-package disaggregation for GPUs is striking the right balance between the degree of composability and the practicality of effectively shifting the burden of high-bandwidth intra-GPU communication from the on-chip to on-package wires.

Figure 5(a) depicts a generic monolithic GPU architecture, with streaming multiprocessors (SMs) and corresponding L1 caches connected to a distributed L2 cache via the GPU network on chip (NoC). L2 cache slices are attached to Memory Controllers (MCs) driving the off-chip DRAM memory interface. While contemporary off-chip DRAM interfaces provide a few TB/s of memory bandwidth (2.7 TB/s in GPU-N), the on-chip interconnects of a modern GPU are designed to transport many tens of TB/s of on-chip bandwidth between the SMs and the on-chip memory hierarchy.

The three possible GPU disaggregation options are shown in Figure 5(b). Option (I), denoted by GPM+MSM(L2+MC), features a GPM containing SMs, L1s, and the GPU NoC, while the MSM includes the L2 cache and the memory controllers. This design can provide more effective die area and die edge than single reticle-limited die to support bigger L2 cache and higher DRAM bandwidth when needed. Option (II), denoted by GPM+MSM(NoC+L2+MC), is similar to (I) but shifts the GPU NoC from the GPM onto the MSM. However, options (I) and (II) are impractical as they both require many tens of $\mathrm{TB} / \mathrm{s}$ of NoC traffic to instead traverse on-package wires, which Section 3.5 later shows is not realizable using current or known proposed technologies.

Thus, we choose option (III), denoted by GPM+MSM(L3+MC), for exploring a practical COPAGPU design as it relies on existing L2 cache bandwidth filtering within the GPM to achieve feasible levels of off-GPM inter-die bandwidth. Additionally, we propose to add an additional layer of L3 cache between the L2 cache and MC, and to have both the L3 cache and the MC implemented on the MSM. Such composable design re-organization can provide more than $4 \times$ higher post-L2 bandwidth using previously proposed package integration technologies.

\subsection{COPA-GPU Architectures and Packaging}

The architectural domain customization in our proposed COPA-GPU is achieved through integration of a GPM with a dedicated domain-optimized MSM using 2.5D or 3D on-package integration that could leverage either planar or vertical die stacking approaches. We consider COPA-GPU architectural options spanning both integration strategies while taking into account the unique interdependency between architectural and package technology choices, distilling the advantages and disadvantages of each approach. 


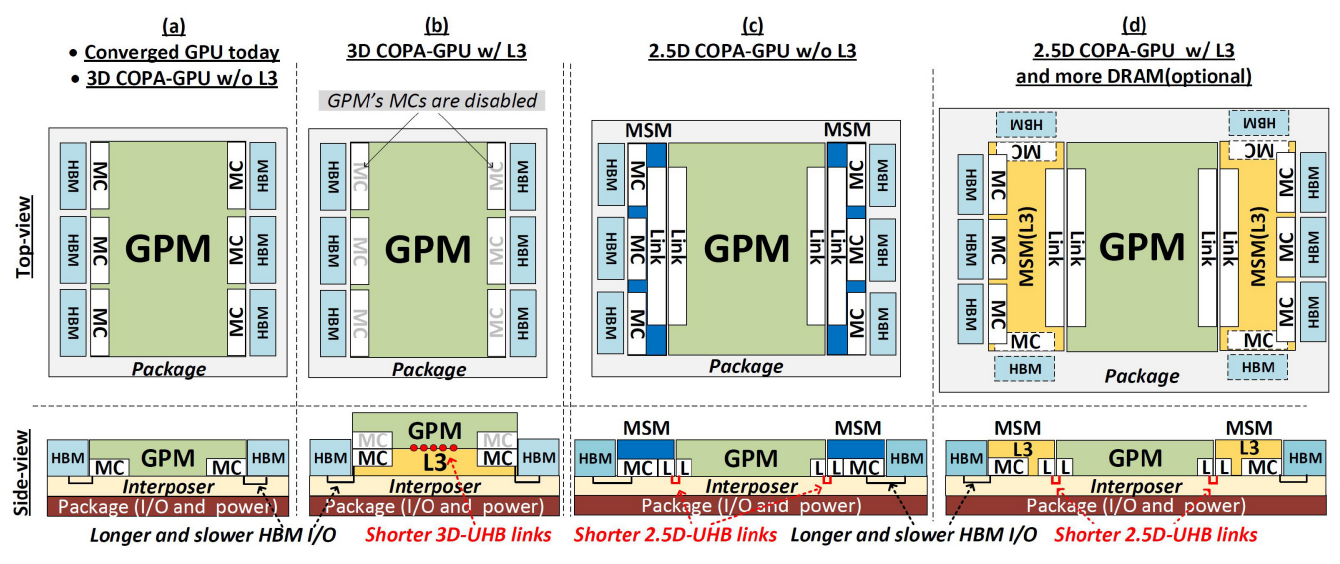

Fig. 6. COPA-GPU architectural options spanning 2.5D and 3D integration domains.

Figure 6(a) shows a high-level top and side view of a modern GPU architecture, including a maximum-sized GPU die with the L2 cache and memory controllers on-die and HBM sites attached via 2.5D on-silicon interposer integration. The same diagram shown in Figure 6(a) also describes the basic COPA-GPU GPM, pre-equipped to be 3D integrated with an MSM die to provide a 3D DL-optimized COPA-GPU variant.

Figure 6(b) illustrates a 3D organization of a DL-optimized COPA-GPU where the GPM die from Figure 6(a) is integrated with an MSM carrying additional L3 cache. The MSM is vertically attached via a 3D ultra-high-bandwidth (3D-UHB) link using high-density intra-die bonding, with up to $14.7 \mathrm{~TB} / \mathrm{s}$ of bandwidth, further detailed in Section 3.5. The MSM is positioned between the GPU and silicon interposer and provides all the essential connectivity between the GPM and the silicon interposer via through silicon vias (TSVs), as shown in Figure 6(b:side-view). The composable nature of a 3D COPA-GPU allows for designs both with (for DL) and without (for HPC) additional L3. The primary disadvantage of a 3D COPA-GPU organization is that it cannot provide any additional die edge to improve DRAM scaling.

Figure 6(c) shows a basic 2.5D COPA-GPU integrating a GPM with up to two MSM modules in a 2.5D organization targeting HPC. The design of a 2.5D COPA-GPU retains most of its original functionality on the base GPM but offloads the memory controllers (MCs) and HBM I/O to new (small) in-package MSMs. A 2.5D COPA-GPU has the advantage that offloading of GPM area allocated to $\mathrm{MC}$ and $\mathrm{HBM} \mathrm{I} / \mathrm{O}$ frees up die area for the implementation of UHB links and additional compute resources in the baseline COPA-GPU.

Figure 6(d) augments the 2.5D COPA-GPU in Figure 6(c) with a large L3 cache and additional DRAM stacks, subject to package area limitations. The same GPM die is reused between Figures 6(c) and 6(d), and this 2.5D DL-optimized organization has several advantages compared to a 3D organization. First, it can provide up to $2 \times$ more L3 capacity than the 3D organization. Second, the additional MSM geometry increases the total available die-edge of the design. This die-edge can provide up to $1.7 \times$ or $2.3 \times$ higher HBM BW and larger capacity, via 10 total HBM sites, or 14 total HBM sites using maximally sized MSMs (not shown).

\subsection{COPA-GPU Microarchitectures}

Figure 7 presents the microarchitecture of the proposed memory systems for both the 3D and 2.5D COPA-GPU designs. Figures 7(a) and 7(b) outline the main components of a 3D COPA-GPU with and without an extended L3 MSM. The most significant difference between current GPU 
$\begin{array}{lll}\text { (a) 3D COPA-GPU w/o L3 } & \text { (b) 3D COPA-GPU w/ L3 }\end{array}$ (HPC-Optimized)

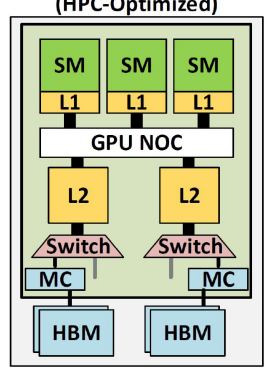

(DL-Optimized)

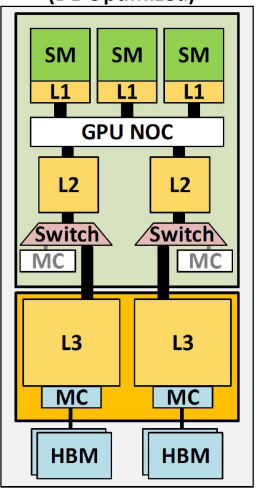

$\begin{array}{lll}\text { (c) 2.5D COPA-GPU w/O L3 } & \text { (d) 2.5D COPA-GPU w/ L3 }\end{array}$

(HPC-Optimized)

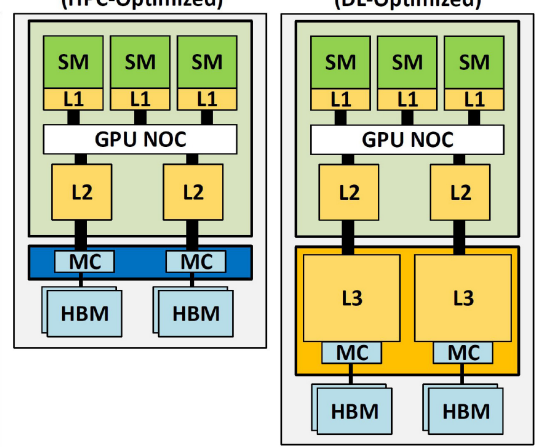

Fig. 7. L3 cache microarchitecture in COPA-GPU designs.

architectures and the 3D COPA-GPU is the addition of a new switching component that steers requests from the L2 to the on-chip memory controller or to the UHB links, depending on whether the MSM is present in-package.

If the MSM is not present (Figure 7(a)), the switch is configured to steer the memory requests directly from the L2 to its local MC, similarly to today's GPUs. If the MSM is present (Figure 7(b)), the switch is reconfigured to steer post-L2 traffic to the L3 via the UHB link. This design requires the MSM to implement its own MC that connects to HBM I/O, with silicon area overheads stemming from adding the UHB link, the TSV-based I/O, and power delivery between GPM and the silicon interposer dies. In the 2.5D COPA-GPU designs without and with additional L3 capacity on the MSM (Figures 7(c) and 7(d)), the GPM is completely stripped of its original MC and HBM $\mathrm{I} / \mathrm{O}$, and post-L2 traffic is always routed to the MSM modules over on-package UHB links. Both of the 2.5D COPA-GPU configurations consist of the same GPM but are equipped with different versions of the MSM to serve different application classes (i.e., with or without L3 and additional HBM sites).

In both designs, the L3 cache is architecturally implemented as yet another level of memoryside cache that (when present) backs the existing L2. It is neither inclusive nor exclusive, nor does it require coherence with the L2 because the L2 already serves as the point of coherence in GPU systems. Lines present in the L2 always supercede lines in the L3 from the GPU's perspective and are written back to the L3 upon eviction from the L2. No requests are routed to the L3 without first being serviced at the L2.

\subsection{Costs and Benefits of the COPA-GPU}

The major drawback of a 2.5D COPA-GPU organization is that it increases the package size. Conversely, the 3D COPA organization has minimal impact on package complexity (same top view), but the base GPM must now account for the implementation of the distributed on-die UHB link bonding used for vertical inter-die communication (Section 3.5) and the TSV overheads used for traversing through the MSM die. We estimate that in the 3D case, the cost of providing as much as $14.7 \mathrm{~TB} / \mathrm{s}$ bandwidth of UHB I/O will consume less than $4 \%$ of silicon surface and metal layers for inter-die communication bonding. The majority of the active area and lower-level metals under the 3D bonding can likely be reused for other logic in the GPM. In 2.5D designs, the maximally sized links result in approximately $6 \%$ area overheads assuming a 20Gbps signaling rate. Section 3.5 provides further details on these assumptions. 
Table 2. Bandwidth and Energy Characteristics Assumptions for 2.5D and 3D Ultra-high Bandwidth (UHB) in This Work

\begin{tabular}{|c|c|c|c|}
\hline Technology & BW Density & Max Bisection BW & Energy/bit \\
\hline \hline $2.5 \mathrm{D}$ & $256 \mathrm{~GB} / \mathrm{s} / \mathrm{mm}$ & $14.7 \mathrm{~TB}$ & $0.3 \mathrm{pJ} / \mathrm{b}$ \\
\hline 3D & $512 \mathrm{~GB} / \mathrm{s} / \mathrm{mm}^{2}$ & $>14.7 \mathrm{~TB}$ & $0.05 \mathrm{pJ} / \mathrm{b}$ \\
\hline
\end{tabular}

Even though both COPA-GPU approaches introduce $4 \%$ to $6 \%$ area overhead due to new MCM communication interfaces, this area (and thus cost overhead) will be more than offset by the savings in the HPC-oriented variants by not carrying forward the unnecessary and expensive DLoriented memory subsystem. Moreover, we believe that in the future even non-composable GPU designs will turn to MCM organizations due to looming reticle limitations, effectively introducing similar MCM link overheads into all GPU designs. The main challenge with such modular GPU designs going forward is its growing integration complexity, effectively shifting focus from on-die integration onto advanced assembly, test, and continuous innovation in on-package interconnect and packaging technologies.

Adding new communication interfaces that memory system requests must traverse, however, comes with an additional energy tax. We estimate that the $2.5 \mathrm{D}$ organized UHB-link consumes less than 9 watts, assuming peak $100 \%$ bandwidth utilization at $0.3 \mathrm{pJ} / \mathrm{b}$ with a $25 \%$ wire toggle-rate. Consequently, the 3D link consumes less than 2 watts, due to its more efficient link technology and overall shorter communication distances (see Section 3.5 for further details).

A large L3 reduces the number of DRAM accesses, more than offsetting the additional cost of the UHB link traversal and L3 accesses. Figure 4 shows that the 960MB and 1.9GB L3 configurations reduce overall DRAM traffic by up to $94 \%$ and $98 \%$, respectively. We estimate that fetching a cache line from an SRAM-based COPA-GPU L3 into the GPM will consume approximately $4 \times$ less energy than accessing HBM memories. These estimates fully account for the energy associated with traveling to an SRAM sub-array on an MSM-die and back, as well as the energy consumed by the SRAM sub-array [9, 11, 41, 77]. Consequently, we estimate that either COPA-GPU design (utilizing a 960MB L3 cache) will reduce the total HBM-related GPU energy consumption by up to 3.4×. While left for future exploration, the cost of the MSM caching modules could be reduced by implementing them in older and less expensive processes.

\subsection{COPA-GPU Enabling Technologies}

The feasibility of COPA-GPUs depends on several technologies reaching maturity for industrial implementation.

Cache Technology Projections: Large caches can be realized through high-density Embedded DRAM (eDRAM) [10] or SRAM technologies [13, 24]. IBM recently implemented a 960MB cache on a $696 \mathrm{~mm}^{2}$ die using a $14 \mathrm{~nm}$ eDRAM technology [10]. Graphcore's recently announced second-generation IPU [43] integrates $896 \mathrm{MB}$ of SRAM along with thousands of cores on a single $823 \mathrm{~mm}^{2}$ die using TSMC's $7 \mathrm{~nm}$ process. Cache density is dictated by a combination of the bitcell area, control overheads, and the bandwidth requirements. Because the COPA-GPU's L3 is designed to provide lower bandwidth than L2, we estimate that a future reticle limited $826-\mathrm{mm}^{2} \mathrm{MSM}$ die (the same die size as an NVIDIA A100 GPU [66]) can provide up to 2GB of L3 cache, though the remainder of this work assumes a conservative projection of a 960MB L3 on an $826 \mathrm{~mm}^{2}$ die, which implies a maximum of 960MB L3 in a 3D COPA-GPU with single MSM die and 1920MB L3 in a 2.5D COPA-GPU with two MSM dies.

High-Bandwidth 2.5D and 3D Interconnects: High-speed links that enable 2.5D integration are rapidly maturing $[19,22,48,76]$. Chen et al. [19] recently demonstrated 20Gbps signaling rates 
Table 3. MLPerf Training and Inference Benchmarks

\begin{tabular}{|l|c|r|r|r|r|}
\hline \multirow{2}{*}{ Benchmark } & \multirow{2}{*}{ Type } & \multicolumn{2}{|c|}{ Small-batch } & \multicolumn{2}{c|}{ Large-batch } \\
\cline { 3 - 6 } & & $\begin{array}{r}\text { Per-GPU } \\
\text { Batch Size }\end{array}$ & $\begin{array}{c}\text { Memory } \\
\text { Footprint }\end{array}$ & $\begin{array}{r}\text { Per-GPU } \\
\text { Batch Size }\end{array}$ & $\begin{array}{c}\text { Memory } \\
\text { Footprint }\end{array}$ \\
\hline resnet & training & 12 & $989 \mathrm{MB}$ & 128 & $6 \mathrm{~GB}$ \\
\hline ssd & training & 4 & $559 \mathrm{MB}$ & 128 & $7.9 \mathrm{~GB}$ \\
\hline maskrcnn & training & 1 & $2.1 \mathrm{~GB}$ & 6 & $9.9 \mathrm{~GB}$ \\
\hline minigo & training & 128 & $105 \mathrm{MB}$ & 2,048 & $1.5 \mathrm{~GB}$ \\
\hline gnmt & training & 32 & $3 \mathrm{~GB}$ & 256 & $8.3 \mathrm{~GB}$ \\
\hline transformer & training & 640 & $4.5 \mathrm{~GB}$ & 5120 & $7.9 \mathrm{~GB}$ \\
\hline ncf & training & 65,526 & $657 \mathrm{MB}$ & $1,048,576$ & $4.5 \mathrm{~GB}$ \\
\hline resnet & inference & 1 & $49 \mathrm{MB}$ & 232 & $1.1 \mathrm{~GB}$ \\
\hline mobilenet & inference & 1 & $16 \mathrm{MB}$ & 704 & $2 \mathrm{~GB}$ \\
\hline ssd-small & inference & 1 & $24 \mathrm{MB}$ & 288 & $2 \mathrm{~GB}$ \\
\hline ssd-large & inference & 1 & $136 \mathrm{MB}$ & 6 & $562 \mathrm{MB}$ \\
\hline gnmt & inference & 1 & $300 \mathrm{MB}$ & 128 & $961 \mathrm{MB}$ \\
\hline
\end{tabular}

Table 4. Detailed GPU Configurations

\begin{tabular}{|l|c|c|c|}
\hline Configuration & NVIDIA V100 & NVIDIA A100 & GPU-N \\
\hline SMs & 80 & 108 & 134 \\
\hline GPU frequency $(\mathrm{GHz})$ & 1.4 & 1.4 & 1.4 \\
\hline FP32 $($ TFLOPS) & 15.7 & 19.5 & 24.2 \\
\hline FP16 (TFLOPS) & 125 & 312 & 779 \\
\hline L2 cache $(\mathrm{MB})$ & 6 & 40 & 60 \\
\hline DRAM BW $(\mathrm{GB} / \mathrm{s})$ & 900 & 1,555 & 2,687 \\
\hline DRAM Capacity $(\mathrm{GB})$ & 16 & 40 & 100 \\
\hline
\end{tabular}

across a $2.5 \mathrm{~mm}$ silicon interposer layer at $0.3 \mathrm{pJ} / \mathrm{b}$, resulting in a $\sim 200 \mathrm{~GB} / \mathrm{s} / \mathrm{mm}$ bandwidth density per layer, which can be further increased at shorter distances. This work assumes a 2.5D COPAGPU UHB link with a bandwidth density of $256 \mathrm{~GB} / \mathrm{s} / \mathrm{mm}$; thus, the dedicated edges of the $826 \mathrm{~mm}^{2}$ GPM module can provide up to $14.7 \mathrm{~TB} / \mathrm{s}$ of off-GPM bandwidth as described in Figure 6(d). While this bandwidth to the MSM far exceeds the actual L3 bandwidth requirement, it provides headroom for future increases in off-die communication.

3D integration is also maturing [14, 29, 31, 33, 79]. For example, TSMC's System on Integrated Chips (SoIC) is expected to provide ultra-dense 3D interconnects [14,29] with greater than $1 \mathrm{~TB} / \mathrm{s} / \mathrm{mm}^{2}$ of inter-die bandwidth, assuming a $1 \mathrm{Gbps}$ signaling rate. We conservatively assume $3 \mathrm{D}-\mathrm{UHB}$ links with $512 \mathrm{~GB} / \mathrm{s} / \mathrm{mm}^{2}$ of bandwidth density at $0.05 \mathrm{pJ} / \mathrm{b}$. Achieving the assumed $14.7 \mathrm{~TB} / \mathrm{s}$ of data bandwidth for a 2.5D COPA-GPU would require $28.7 \mathrm{~mm}^{2}$ (less than $4 \%$ ) of silicon area for the inter-die communication bonding. Table 2 summarizes the UHB link characteristics for both $2.5 \mathrm{D}$ and $3 \mathrm{D}$ scenarios.

\section{EVALUATION}

We now present our simulation methodology, examine the performance sensitivity of the baseline COPA-GPU architecture to DRAM bandwidth and on-package LLC capacity, and evaluate several specific DL-specialized COPA-GPU configurations in both training and inference scenarios. 


\subsection{Methodology}

To provide performance projections for DL workloads, we perform our studies using unmodified workloads from the MLPerf training and inference benchmark suites [53, 70] for one end-to-end iteration of the workloads, rather than using disjoint and isolated GPU kernel calls. This approach allows us to characterize the overall throughput and capture inter-layer activation and weight reuse in the L3 that will drive future memory system design on GPUs. MLPerf is the de facto standard for DL benchmarking and is maintained by major DL chip vendors including NVIDIA, Google, and Intel. Table 3 shows the seven MLPerf training benchmarks and the five inference benchmarks used, taken from NVIDIA's publicly available MLPerf training v0.6 and MLPerf inference v0.5 submissions [56, 57]. These codes are highly optimized for NVIDIA GPUs by exploiting high-throughput Tensor Cores [64] and have demonstrated performance scalability from a single to thousands of GPUs.

To build a complete picture of future deep learning scenarios, we run all our DL benchmarks in two different configurations. For DL training, we use a large per-GPU batch size to characterize a single-GPU training situation and a small per-GPU batch size to represent a large-scale training system. For DL inference, we use a large per-GPU batch size to represent a datacenter processing inference task with large numbers of concurrent queries and a small per-GPU batch size for a latency-sensitive scenario with real-time processing requirements. Although small-batch inference workloads are less common on the large datacenter-grade GPUs we evaluate in this article, we include them in our studies for completeness as future GPU designs could be scaled down (in aggregate) to build niche-specific GPU products in traditional or COPA-GPU form, and also there has been a lot of recent work $[16,17,21,80]$ focusing on efficient small-batch inference for latency-sensitive DL workloads.

The batch sizes we choose for each scenario are taken from NVIDIA's MLPerf submissions and are shown in Table 3. For our evaluations, we execute each application on a single NVIDIA Tesla V100 and collect a GPU execution trace from a full end-to-end iteration. We focus on the perGPU workload analysis and omit the all-reduce synchronization overheads when projecting performance for large-scale DL training. All-reduce performance largely depends on the implementation of the inter-GPU network, which is beyond the scope of this work but is also receiving attention within the architecture community $[42,45]$.

We simulate these workloads using a modified version of NVIDIA's NVArchSim [78]. NVArchSim provides very fast simulation of deterministic portions of an application without sacrificing simulation accuracy or correctness during non-deterministic application phases and has high accuracy for the MLPerf workloads we focus on in this work. Our analysis focuses on the projected GPU-N configuration described in Table 4. GPU-N's SM count growth beyond NVIDIA A100's is proportional to the projected FP32 throughput (from Table 1) with a constant SM frequency. GPUN's DRAM capacity is projected based on a linear extrapolation similar to the projections done for the DRAM bandwidth.

\subsection{Performance Sensitivity to DRAM Bandwidth}

We perform a detailed sweep of the DRAM bandwidth settings for the basic COPA-GPU design without L3 (similar to GPU-N) from half of its nominal bandwidth (1.3TB/s) up through infinite bandwidth. Figure 8 summarizes the overall performance normalized to the $2.7 \mathrm{~TB} / \mathrm{s}$ baseline. Workload performance scales steadily with growing DRAM bandwidth up to a $3 \times \mathrm{BW}(8.1 \mathrm{~TB} / \mathrm{s})$ setting in most of the training and inference scenarios, with diminishing returns beyond this point. Smallbatch inference applications are less sensitive to DRAM bandwidth due to their relatively small footprint (more details in Section 4.3), while the remaining DL training and inference workloads 


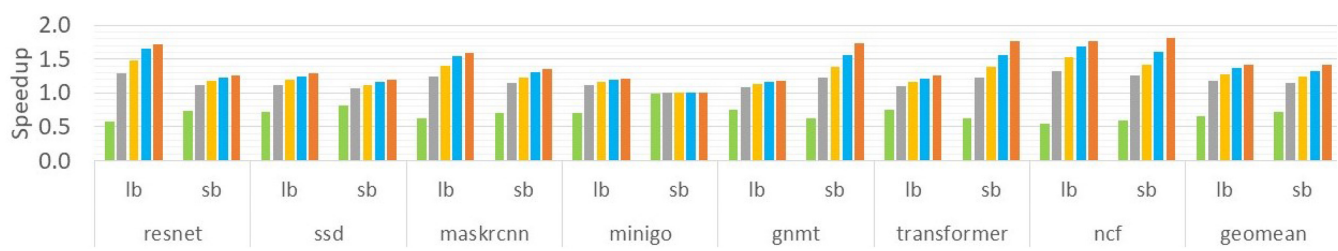

(a) Training

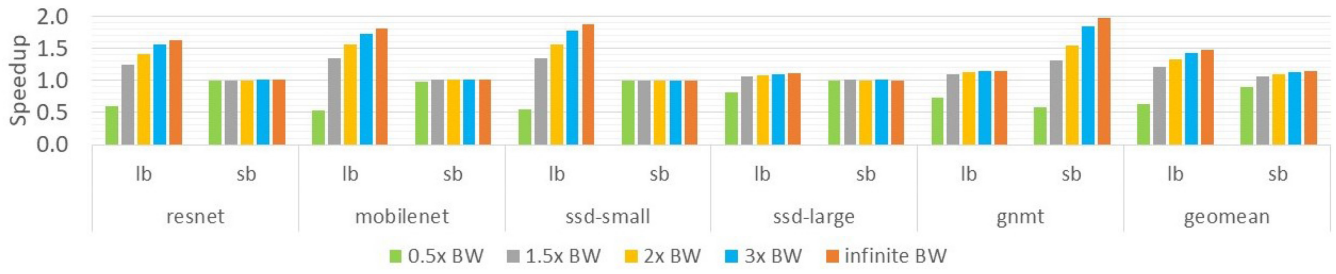

(b) Inference

Fig. 8. Performance of a basic COPA-GPU with varying DRAM bandwidth for large-batch $(l b)$ and smallbatch $(s b)$ settings, normalized to the baseline GPU-N performance.

show that even a relatively modest $1.5 \times$ increase in DRAM bandwidth from $2.7 \mathrm{~TB} / \mathrm{s}$ to $4 \mathrm{~TB} / \mathrm{s}$ would lead to notable speedups of up to $18 \%$ for training and $21 \%$ for inference.

We notice that some applications are more sensitive to DRAM bandwidth at small-batch scenarios, while others are more sensitive at large-batch scenarios. There are multiple factors that determine the sensitivity of an application to DRAM bandwidth as the batch size changes. In general, smaller batch sizes mean less data reuse at the batch dimension, thereby lowering the compute intensity (FLOPS/byte) of the application and making it more memory-bound. As a result, smallbatch settings would be more sensitive to DRAM bandwidth. However, smaller batch sizes could also result in further SM underutilization as shown in Figure 2, which would consequently lead to a lower DRAM bandwidth pressure. In addition, for applications with small memory footprint (as shown in Table 3), smaller batch sizes also allow a significantly larger portion of the model data (activations and weights) to be stored on-chip, reducing the DRAM bandwidth pressure. The sensitivity of an application to bandwidth is impacted by all these factors and thus can vary across different applications.

From these results we conclude that modern GPUs are on track to be severely DRAM bandwidth limited and that additional raw DRAM bandwidth is the most powerful tool for scaling DL performance. However, because evolutionary improvement in HBM memory frequency and pin density is unlikely to provide a substantially higher bandwidth scaling ratio than our aggressively projected $1.7 \times$ (for GPU-N), we explore the potential of a very large- and high-bandwidth on-package cache to close this gap in future COPA-GPUs.

\subsection{Performance Sensitivity to Cache Capacity}

To understand the impact of large caches on DRAM bandwidth reduction in future GPUs, we sweep the LLC (L2) cache capacity of the basic COPA-GPU without L3 from 60MB to 3,840MB. A perfect L2, where all requests hit in the L2 caches, serves as the upper bound on performance. Figure 9 shows the performance of these different L2 configurations, normalized to the $60 \mathrm{MB}$ baseline. Increasing the L2 capacity results in performance gain equivalent to large increases in DRAM bandwidth, primarily because of the significant off-chip DRAM traffic reduction, as shown in Figure 4. 


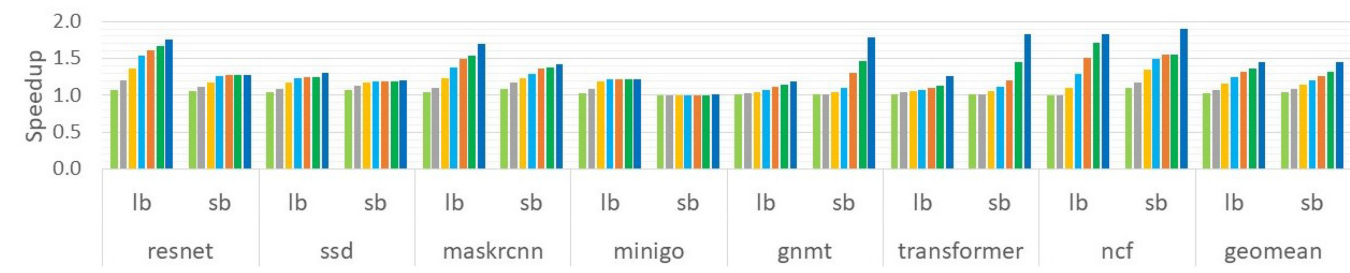

(a) Training

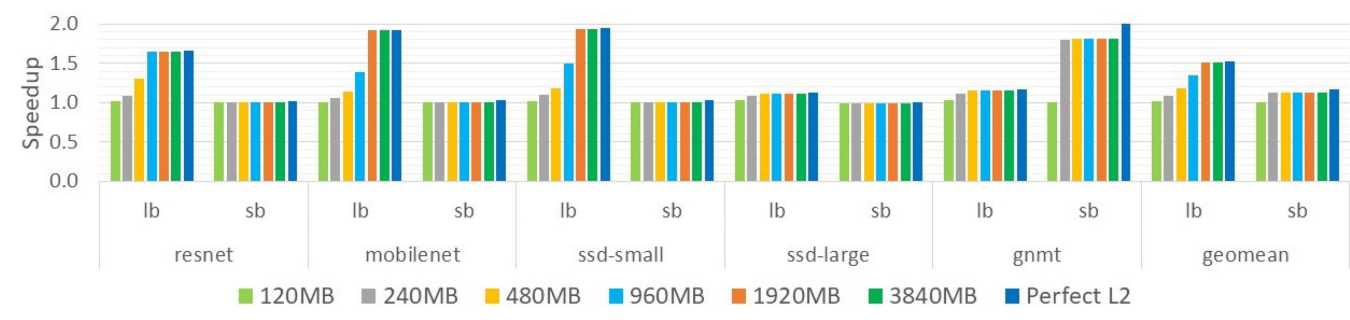

(b) Inference

Fig. 9. Performance of a basic COPA-GPU with varying LLC capacities for both large-batch $(l b)$ and smallbatch $(s b)$ settings, normalized to the baseline GPU-N performance.

Table 5. COPA-GPU Architectural Parameters

\begin{tabular}{|l|c|c|c|}
\hline $\begin{array}{l}\text { Architecture } \\
\text { Configuration }\end{array}$ & $\begin{array}{c}\text { LLC Capacity } \\
(\mathrm{MB})\end{array}$ & $\begin{array}{c}\text { DRAM BW } \\
\text { (TB/s) }\end{array}$ & $\begin{array}{c}\text { DRAM Capacity } \\
(\mathrm{GB})\end{array}$ \\
\hline GPU-N & 60 & 2.7 & 100 \\
\hline COPA-GPU-1 & 960 & 2.7 & 100 \\
\hline COPA-GPU-2 & 960 & 4.5 & 167 \\
\hline COPA-GPU-3 & 1,920 & 2.7 & 100 \\
\hline COPA-GPU-4 & 1,920 & 4.5 & 167 \\
\hline COPA-GPU-5 & 1,920 & 6.3 & 233 \\
\hline Perfect L2 & infinite & infinite & infinite \\
\hline
\end{tabular}

For DL training, $480 \mathrm{MB}$ of L2 performs slightly worse than a $1.5 \times$ increase in DRAM bandwidth, and $960 \mathrm{MB}$ of L2 performs slightly worse than a $2 \times$ increase in DRAM bandwidth (comparing to Figure 8). Yet larger L2 caches continue to incrementally improve the performance, but even a $3,840 \mathrm{MB}$ L2 still results in an $8 \%$ and $13 \%$ performance gap in large-batch and small-batch DL training workloads when compared to a perfect L2. Because DL inference applications generally have smaller memory footprint sizes than DL training, performance saturates once all workload data can be cached. The saturation points are 1,920MB and 240MB for large- and for small-batch inference, respectively, which correspond to their memory footprints listed in Table 3.

We conclude that a substantially larger LLC is an attractive solution for scaling DL performance within a COPA-GPU; however, closing the performance gap to a perfect L2 would require 4GB of on-package cache, which is impractical even under aggressive technology projections. As a result, to maximize DL performance, COPA-GPUs must not just utilize a very large L3 cache but also combine a large L3 and higher DRAM bandwidth made available with additional MSM-die edge in a $2.5 \mathrm{D}$ COPA-GPU design. 


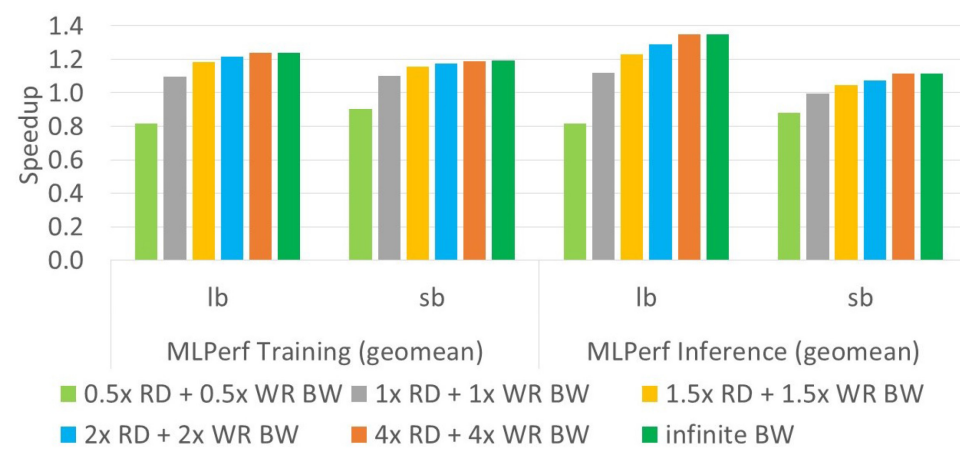

Fig. 10. Performance of a COPA-GPU-1 with varying UHB link bandwidth to the L3 cache, normalized to the baseline GPU-N performance.

\subsection{Performance Scaling with COPA-GPU}

Table 5 summarizes the DL-optimized COPA-GPU configurations that are enabled by architectural choices outlined in Section 3. The COPA-GPU-1 design provides 960MB of L3 cache through either 3D stacking with a single maximum-sized MSM die or 2.5D stacking with two MSM dies, each set to half of the maximum die size. The COPA-GPU-2 option with more HBM resources applies only to $2.5 \mathrm{D}$, by exploiting the extra MSM die edge area to provide a $1.7 \times$ higher HBM bandwidth and a larger HBM capacity (10 HBM sites in total). With two maximally sized MSM dies in the 2.5D stacking scenario, the L3 cache capacity and the additional DRAM bandwidth can be scaled to up to $1,920 \mathrm{MB}$ and $2.3 \times$ of the baseline DRAM bandwidth (via $14 \mathrm{HBM}$ sites), respectively. As a result, we consider three additional COPA-GPU configurations with 1,920MB of L3 cache and varying HBM resources: COPA-GPU-3, COPA-GPU-4, and COPA-GPU-5, with 2.7TB/s, 4.5TB/s, and 6.3TB/s of DRAM bandwidth, respectively.

Before moving to overall results, we first analyze the UHB on-package link bandwidth requirements by sweeping the unidirectional L3 bandwidth in the COPA-GPU-1 configuration with half of its baseline DRAM bandwidth $(0.5 \times \mathrm{RD}+0.5 \times \mathrm{WR}$, which sums to $2.7 \mathrm{~TB} / \mathrm{s})$ up through infinite bandwidth. Figure 10 summarizes the geometric mean speedup of the MLPerf applications (training and inference), normalized to the baseline GPU-N (without L3). The performance increases substantially when the L3 bandwidth grows from $0.5 \times \mathrm{RD}+0.5 \times \mathrm{WR}$ to $2 \times \mathrm{RD}+2 \times \mathrm{WR}$, with diminishing returns beyond that point. Overall, the UHB bandwidth configuration of $2 \times \mathrm{RD}+2 \times$ WR (total $10.8 \mathrm{~TB} / \mathrm{s}$ ) comes within $3 \%$ and $6 \%$ of the unlimited bandwidth settings for training and inference, respectively. This bandwidth is well within the capabilities of next-generation $2.5 / 3 \mathrm{D}$ interconnect technologies assumed in our designs.

We also evaluate the effect of the UHB link latency by varying the total round-trip latency between the L2 and L3 caches from $0.25 \times$ to $1 \times$ of the DRAM access latency. Our experiments (not shown) indicate that COPA-GPU architecture is not sensitive to L3 latency, as performance changes by less than $2 \%$ across this latency range. As a result, we set the UHB link bandwidth to be $2 \times \mathrm{RD}+2 \times \mathrm{WR}$, for a total of $10.8 \mathrm{~TB} / \mathrm{s}$ and the round-trip latency between L2 and L3 (UHB link latency plus L3 access latency) to be half of the DRAM access latency for all the COPA-GPU configurations with L3 that are listed in Table 5.

Figure 11 summarizes the MLPerf training and inference performance of the COPA-GPU designs from Table 5 in large batch $(l b)$ and small batch $(s b)$ settings. Unsurprisingly, larger cache capacity and higher memory bandwidth universally improve both large-batch and small-batch training performance. For example, the additional cache capacity in COPA-GPU-1 improves large-batch and small-batch performance by $21 \%$ and $18 \%$, respectively, making it an attractive 3D COPA-GPU 


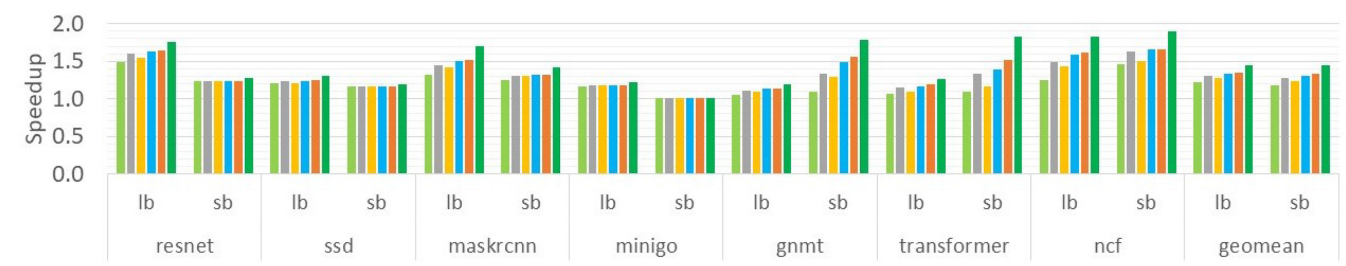

(a) Training

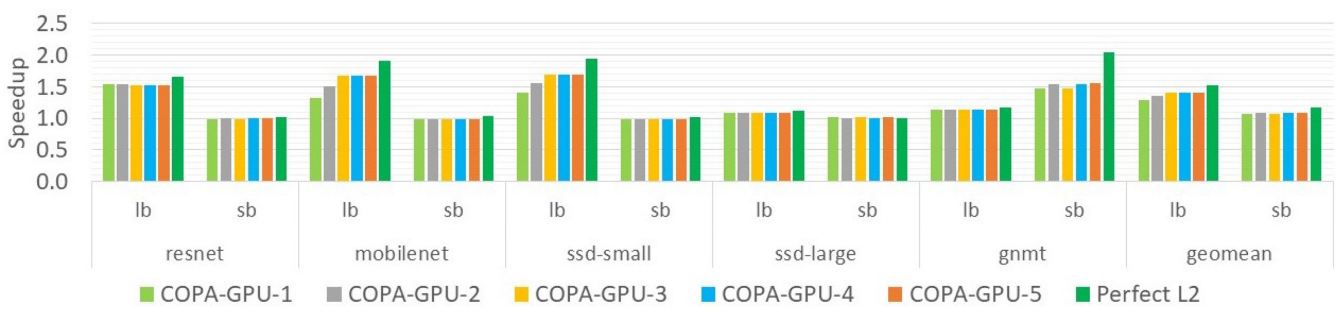

(b) Inference

Fig. 11. Performance of DL-optimized COPA-GPUs with varying designs, normalized to the baseline GPU-N performance. Large-batch and small-batch cases are denoted by $l b$ and $s b$.

design. Further, the additional HBM bandwidth provided by the COPA-GPU-2 configuration achieves an overall $31 \%$ and $27 \%$ speedup, making it an attractive 2.5D COPA-GPU design choice. COPA-GPU-3 doubles L3 capacity instead of scaling DRAM bandwidth and results in lower speedups than COPA-GPU-2 (by 4\%) at both large- and small-batch scenarios, indicating that increasing L3 capacity alone is not the best approach for scaling DL training performance. Finally, ambitious DRAM options such as COPA-GPU-4 and COPA-GPU-5 result in marginal performance gains $(4 \%)$ when compared to COPA-GPU-2 and do not justify the additional costs of these systems.

For large-batch inference, the large L3 capacity in COPA-GPU-1 improves the performance by $29 \%$ and COPA-GPU-3 can reach speedup as high as $40 \%$. With 1,920MB of L3, further increases in HBM bandwidth are not beneficial because most of the DRAM traffic has already been filtered out by the large L3 cache. In fact, though not shown, we find that DRAM bandwidth could be even reduced by $50 \%$ without affecting the performance. For small-batch inference, because the performance saturation point is at $240 \mathrm{MB}$ of LLC, the performance improvement via the combination of L3 and HBM is just $9 \%$.

Overall, we conclude that COPA-GPU-2, which combines a substantially larger L3 cache (960MB) and moderately higher DRAM bandwidth $(4.5 \mathrm{~TB} / \mathrm{s})$, is likely the most optimal COPA-GPU design that will perform well for both DL training (with $31 \%$ gain at large-batch and $27 \%$ gain at smallbatch) and inference (with 35\% gain at large-batch and $8 \%$ gain at small-batch) without adding significant unnecessary cost through overprovisioning of memory resources. Inference is more sensitive to cache capacity and less sensitive to DRAM bandwidth than DL training, and trading off additional HBM resources for increased LLC capacity could be a plausible strategy if designing a COPA-GPU specialized for just DL inference.

A large COPA-GPU-enabled L3 reduces the total DRAM-related per-GPU energy consumption by up to 3.4×, as shown in Section 3.3. However, the improved DL-optimized COPA-GPU utilization may lead to increased total design power that may not be entirely mitigated by the power reduction within the memory system. To mitigate growing thermal density, we expect future high-end GPU systems will rely on liquid cooling technologies to enable increased thermal envelopes compared to those possible today $[18,67]$. 


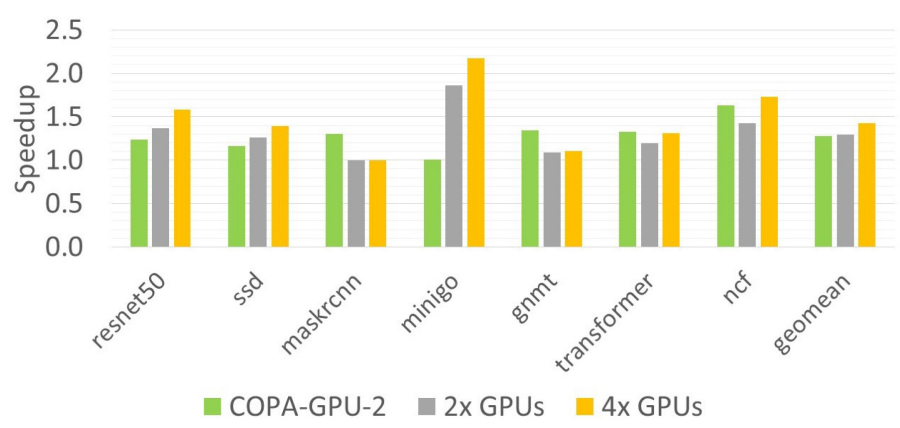

Fig. 12. Performance of DL-optimized COPA-GPU-2 designs versus additional data parallelism via $2 \times \mathrm{GPU}$ Ns and $4 \times$ GPU-Ns, normalized to the baseline GPU-N performance.

\subsection{COPA-GPU Training Cost Efficiency at Scale}

At scale, multi-GPU training performance is eventually limited by decreasing per-GPU compute efficiency and growing system-level synchronization overheads. To understand the scale-out efficiency of DL-optimized COPA-GPUs, we compare the performance of a COPA-GPU-2-based system with $2 \times$ and $4 \times$ larger systems composing the baseline GPU-Ns. We fix the global batch size across all configurations to maintain the same statistical efficiency [68]. Consequently, the perGPU batch size drops to one-half and one-quarter for the baseline GPU-N configurations. We do not extrapolate the additional overheads of distributed gradient synchronization at larger scales, and thus our analysis favors the $2 \times$ and $4 \times$ GPU-N configurations.

Figure 12 shows that doubling and quadrupling the number of baseline GPU-N instances $(2 \times$ GPU-Ns and $4 \times$ GPU-Ns) results in mean $29 \%$ and $43 \%$ performance gains, respectively, for our training workloads. We find that a DL-optimized COPA-GPU-2 configuration (with $27 \%$ performance gain) provides similar levels of performance to $2 \times$ GPU-Ns yet should cost significantly less than buying and hosting $2 \times$ larger installations of traditional GPU-Ns. Notice that at smallbatch setting, maskrcnn has already reached the minimal batch size of 1 such that further data parallelism would not help. In general, applications that are less memory-bound and exploit more internal parallelism would benefit more from data parallelism.

When compared to GPU-N, even though COPA-GPU-2 doubles the area by adding close to $826 \mathrm{~mm}^{2}$ of silicon, it is dominated by regular SRAM cache arrays that are expected to achieve high manufacturing yields due to built-in redundancy and error recovery. Moreover, this aggregate area is split into two smaller dies, resulting in substantially lower cost per $\mathrm{mm}^{2}$. COPA-GPU-2 integrates $1.6 \times$ more HBM memory resources, resulting in total aggregate cost lower than $2 \times$ of GPU-N. Thus, DL-optimized COPA-GPUs will provide substantially better cost performance at scale, saving on not just overall GPU cost but additional system-level collateral such as datacenter floorspace, CPUs, network switches, and other peripheral devices.

\section{RELATED WORK}

Leveraging high-capacity and high-bandwidth on-chip and on-wafer caches, or scratch memories, to store DL weights and activations has been well explored for DL training/inference architectures. DaDianNao [15] was designed with $36 \mathrm{MB}$ of on-chip eDRAM to cache model weights. Google's TPUv1 [38] allocated 28MB of on-chip memory mostly for caching activations, while TPUv2 and TPUv3 [37] increased it to 32MB, and then TPUv4i [36] further increased it to 144MB. Recent DL accelerators such as Graphcore's IPU [34, 43], Groq's TSP [25], Alibaba's HanGuang [35], and Cerebras's WSE $[46,47]$ replaced off-chip memory with hundreds of MBs of high-bandwidth 
on-chip SRAM to satisfy increasing memory bandwidth requirements. Unlike domain-specific accelerators that are highly tuned for DL workloads, COPA-GPUs provide high levels of GPU design reuse across application domains, while also enabling memory-system specialization for individual domains.

Multi-chip module (MCM) packaging has been extensively studied and deployed to integrate heterogeneous and homogeneous chips within a package, aiding the scaling of compute and memory bandwidths for a wide variety of legacy GPU and CPU applications. Prior work $[8,9,55]$ has focused on developing MCM-GPU architectures to strong scale GPU performance beyond the limitations of a single monolithic die by leveraging on-package and on-board integration technologies. In a follow-on work [71,81], the authors extend MCM-GPU architectures with advanced caching and HW/SW cache-coherency protocols to overcome NUMA limitations. MCM-3D-NoC [69] tackled the interconnect scalability issues of MCM integration over active interposers. In the CPU space, recent AMD CPU architectures $[12,59]$ leverage multi-module on-board integration to provide scalable and modular CPU architectures. Finally, Kannan et al. [39] proposed to disaggregate large monolithic CPU designs into smaller chips for cost reduction. In [74], the authors propose and quantify the costs and benefits of using MCMs with fine-grained domain-specific chiplets for DL inference.

General purpose CPU SoCs have already leveraged MCM designs with very large on-package eDRAM caches. Intel improved its mobile CPU performance by combining a CPU SoC and 128MB of on-package eDRAM organized as a victim cache [32], and IBM scaled up its Gen-Z mainframe performance with $960 \mathrm{MB}$ of L4 eDRAM cache [10].

While a COPA-GPU leverages the previously proposed concepts of on-package integration and large caches, our work is (1) the first to identify and solve the diverging architectural requirements between FP32 (or larger)-based HPC and FP16 (or smaller)-based DL workloads in GPUs and (2) the first to develop the reusable GPU architecture concept, enabling cost-effective GPU domain specialization for HPC and deep learning.

\section{CONCLUSION}

In this work, we demonstrate that diverging architectural requirements between the HPC and DL application domains put converged GPU designs on a trajectory to become significantly underprovisioned for DL and over-provisioned for HPC. We propose a new composable GPU architecture that leverages emerging circuit and packaging technologies to provide specialization, while maintaining substantial compatibility across product lines. We demonstrate that COPA-GPU architectures can enable selective deployment of on-package cache and off-chip DRAM resources, allowing manufacturers to easily tailor designs to individual domains. Our analysis shows that DL-optimized COPA-GPUs will provide impressive per-GPU training and inference performance improvements, while still efficiently supporting scaled-down HPC-targeted designs. DL-optimized COPA-GPUs will also result in reduced datacenter cost by minimizing the number of GPUs required to achieve scale-out training performance targets, making COPA-GPU an attractive paradigm for increasing individual and aggregate GPU performance without over-optimizing the product for any specific domain.

\section{REFERENCES}

[1] Mark James Abraham, Teemu Murtola, Roland Schulz, Szilárd Páll, Jeremy C. Smith, Berk Hess and Erik Lindahl. 2015. GROMACS: High performance molecular simulations through multi-level parallelism from laptops to supercomputers. SoftwareX 1-2 (2015), 19-25.

[2] Sjors H.W. Scheres. 2012. RELION: Implementation of a Bayesian approach to cryo-EM structure determination. Fournal of Structural Biology 180, 3 (2012), 519-530. 
[3] Dimitri Komatitsch. 2011. Fluid.solid coupling on a cluster of GPU graphics cards for seismic wave propagation. Comptes Rendus Mécanique 339, 2-3, 125-135.

[4] Romelia Salomon-Ferrer, David A. Case and Ross C. Walker. 2013. An overview of the Amber biomolecular simulation package. WIREs Comput Mol. Sci. 3, 2, 198-210.

[5] Johnathan Alsop, Matthew D. Sinclair, Rakesh Komuravelli, and Sarita V. Adve. 2016. GSI: A GPU stall inspector to characterize the sources of memory stalls for tightly coupled GPUs. In International Symposium on Performance Analysis of Systems and Software (ISPASS'16). 172-182.

[6] AMD. 2020. AMD INSTINCT ${ }^{T M}$ MI100 ACCELERATOR. https://www.amd.com/system/files/documents/instinctmi100-brochure.pdf.

[7] Dario Amodei, Sundaram Ananthanarayanan, Rishita Anubhai, Jingliang Bai, Eric Battenberg, Carl Case, Jared Casper, Bryan Catanzaro, Qiang Cheng, Guoliang Chen, Jie Chen, Jingdong Chen, Zhijie Chen, Mike Chrzanowski, Adam Coates, Greg Diamos, Ke Ding, Niandong Du, Erich Elsen, Jesse Engel, Weiwei Fang, Linxi Fan, Christopher Fougner, Liang Gao, Caixia Gong, Awni Hannun, Tony Han, Lappi Johannes, Bing Jiang, Cai Ju, Billy Jun, Patrick LeGresley, Libby Lin, Junjie Liu, Yang Liu, Weigao Li, Xiangang Li, Dongpeng Ma, Sharan Narang, Andrew Ng, Sherjil Ozair, Yiping Peng, Ryan Prenger, Sheng Qian, Zongfeng Quan, Jonathan Raiman, Vinay Rao, Sanjeev Satheesh, David Seetapun, Shubho Sengupta, Kavya Srinet, Anuroop Sriram, Haiyuan Tang, Liliang Tang, Chong Wang, Jidong Wang, Kaifu Wang, Yi Wang, Zhijian Wang, Zhiqian Wang, Shuang Wu, Likai Wei, Bo Xiao, Wen Xie, Yan Xie, Dani Yogatama, Bin Yuan, Jun Zhan, and Zhenyao Zhu. 2016. Deep Speech 2: End-to-End speech recognition in english and mandarin. In International Conference on Machine Learning (ICML'16).

[8] Akhil Arunkumar, Evgeny Bolotin, Benjamin Cho, Ugljesa Milic, Eiman Ebrahimi, Oreste Villa, Aamer Jaleel, CaroleJean Wu, and David Nellans. 2017. MCM-GPU: Multi-Chip-Module GPUs for continued performance scalability. In International Symposium on Computer Architecture (ISCA'17).

[9] Akhil Arunkumar, Evgeny Bolotin, David Nellans, and Carole-Jean Wu. 2019. Understanding the future of energy efficiency in multi-module GPUs. In International Symposium on High-Performance Computer Architecture (HPCA'19)

[10] Christopher Berry, Brian Bell, Adam Jatkowski, Jesse Surprise, John Isakson, Ofer Geva, Brian Deskin, Mark Cichanowski, Dina Hamid, Chris Cavitt, Gregory Fredeman, Anthony Saporito, Ashutosh Mishra, Alper Buyuktosunoglu, Tobias Webel, Preetham Lobo, Pradeep Parashurama, Ramon Bertran, Dureseti Chidambarrao, David Wolpert, and Brandon Bruen 2020. IBM z15: A 12-Core 5.2GHz Microprocessor. In International Solid-State Circuits Conference (ISSCC'20).

[11] Evgeny Bolotin, David Nellans, Oreste Villa, Mike O’Connor, Alex Ramirez, and Steve Keckler. 2015. Designing efficient heterogeneous memory architectures. IEEE Micro 35, 4 (2015), 60-68.

[12] Thomas Burd, Noah Beck, Sean White, Milam Paraschou, Nathan Kalyanasundharam, Gregg Donley, Alan Smith, Larry Hewitt, and Samuel Naffziger. 2019. 'Zeppelin': An SoC for multichip architectures. IEEE fournal of Solid-State Circuits 54, 1 (January 2019), 133-143.

[13] J. Chang, Y. Chen, G. Chan, H. Cheng, P. Wang, Y. Lin, H. Fujiwara, R. Lee, H. Liao, P. Wang, G. Yeap, and Q. Li. 2020. A 5nm 135Mb SRAM in EUV and high-mobility-channel FinFET technology with metal coupling and chargesharing write-assist circuitry schemes for high-density and low-VMIN applications. In International Solid-State Circuits Conference (ISSCC'20).

[14] Ming-Fa Chen, Fang-Cheng Chen, Wen-Chih Chiou, and Doug C. H. Yu. 2019. System on Integrated Chips (SoIC) for 3D heterogeneous integration. In Electronic Components and Technology Conference (ECTC'19).

[15] Yunji Chen, Tao Luo, Shaoli Liu, Shijin Zhang, Liqiang He, Jia Wang, Ling Li, Tianshi Chen, Zhiwei Xu, Ninghui Sun, and Olivier Temam. 2014. DaDianNao: A machine-learning supercomputer. In International Symposium on Microarchitecture (MICRO'14).

[16] Yujeong Choi, Yunseong Kim, and Minsoo Rhu. 2021. Lazy Batching: An SLA-aware batching system for cloud machine learning inference. In International Symposium on High-Performance Computer Architecture (HPCA'21). 493-506.

[17] Yujeong Choi and Minsoo Rhu. 2020. PREMA: A predictive multi-task scheduling algorithm for preemptible neural processing units. In International Symposium on High-Performance Computer Architecture (HPCA'20). 220-233.

[18] DataCeneter Knowledge 2018. Google Brings Liquid Cooling to Data Centers to Cool Latest AI Chips. https://www. datacenterknowledge.com/google-alphabet/google-brings-liquid-cooling-data-centers-cool-latest-ai-chips.

[19] Behzad Dehlaghi, Nijwm Wary, and Tony Carusone. 2019. Ultra-short-reach interconnects for die-to-die links: Global bandwidth demands in microcosm. IEEE Solid-State Circuits Magazine 11, 2 (2019), 42-53.

[20] Jacob Devlin, Ming-Wei Chang, Kenton Lee, and Kristina. Toutanova. 2018. BERT: Pre-training of deep bidirectional transformers for language understanding. In arXiv preprint arXiv:1810.04805

[21] Pin Gao, Lingfan Yu, Yongwei Wu, and Jinyang Li. 2018. Low latency RNN inference with cellular batching. In Proceedings of the 13th EuroSys Conference (EuroSys'18).

[22] David Greenhill, Ron Ho, David Lewis, Herman Schmit, Kok Chan, Sean Atsatt, Dana How, Peter McElheny, Keith Duwel, Jeffrey Schulz, Darren Faulkner, Gopal Iyer, George Chen, Hee Phoon, Han Lim, Wei-Yee Koay, and Ty 
Garibay. 2017. A 14nm 1GHz FPGA with 2.5D Transceiver Integration. In International Solid-State Circuits Conference (ISSCC'17).

[23] Shixiang Gu, Ethan Holly, Timothy Lillicrap, and Sergey Levine. 2017. Deep reinforcement learning for robotic manipulation with asynchronous off-policy updates. In International Conference on Robotics and Automation (ICRA'17).

[24] Zheng Guo, Daeyeon Kim, Satyanand Nalam, Jami Wiedemer, Xiaofei Wang, and Eric Karl. 2018. A 23.6-Mb/mm2 SRAM in 10-nm FinFET technology with pulsed-pMOS TVC and stepped-WL for low-voltage applications. In International Solid-State Circuits Conference (ISSCC'18).

[25] Linley Gwennap. 2020. Groq rocks neural networks. In Microprocessor Report by the Linley Group.

[26] Kaiming He, Xiangyu Zhang, Shaoqing Ren, and Jian Sun. 2016. Deep residual learning for image recognition. In Conference on Computer Vision and Pattern Recognition (CVPR'16).

[27] Xiangnan He, Lizi Liao, Hanwang Zhang, Liqiang Nie, Xia Hu, and Tat-Seng Chua. 2017. Neural collaborative filtering. In International World Wide Web Conference (WWW'17).

[28] S. Y. Hou, W. C. Chen, C. Hu, C. Chiu, K. C. Ting, T. S. Lin, W. H. Wei, W. C. Chiou, V. J. C. Lin, V. C. Y. Chang, C. T. Wang, C. H. Wu, and D. Yu. 2017. Wafer-Level integration of an advanced logic-memory system through the second-generation CoWoS technology. IEEE Transactions on Electron Devices 64, 10 (2017), 4071-4077.

[29] C. C. Hu, M. F. Chen, W. C. Chiou, and Doug Yu. 2019. 3D Multi-chip integration with system on integrated chips (SoIC). In Symposium on VLSI Technology.

[30] Itay Hubara, Matthieu Courbariaux, Daniel Soudry, Ran El-Yaniv, and Yoshua Bengio. 2016. Binarized neural networks. In Advances in Neural Information Processing Systems 29.

[31] D. Ingerly, K. Enamul, W. Gomes, D. Jones, K. Kolluru, A. Kandas, G.-S Kim, H. Ma, D. Pantuso, C. F. Petersburg, M. Phen-givoni, A. Pillai, A. Sairam, P. Shekhar, P. Sinha, P. Stover, A. Telang, Z. Zell, and R. Criss. 2019. Foveros: 3D Integration and the use of face-to-face chip stacking for logic devices. In IEEE International Electron Devices Meeting (IEDM’19).

[32] Intel. 2015. $14 \mathrm{~nm}$ Technology Announcement. https://www.intel.com/content/dam/www/public/us/en/documents/ presentation/advancing-moores-law-in-2014-presentation.pdf.

[33] Intel. 2019. Lakefield: Hybrid cores in 3D package. https://www.hotchips.org/hc31/HC31_2.10_LKF_HC_2019_Final_ v7.pdf.

[34] Zhe Jia, Blake Tillman, Marco Maggioni, and Daniele Paolo Scarpazza. 2019. Dissecting the graphcore IPU architecture via microbenchmarking. In arXiv preprint arXiv:1912.03413.

[35] Yang Jiao, Liang Han, Rong Jin, Yi-Jung Su, Chiente Ho, Li Yin, Yun Li, Long Chen, Zhen Chen, Lu Liu, Zhuyu He, Yu Yan, Jun He, Jun Mao, Xiaotao Zai, Xuejun Wu, Yongquan Zhou, Mingqiu Gu, Guocai Zhu, Rong Zhong, Wenyuan Lee, Ping Chen, Yiping Chen, Weiliang Li, Deyu Xiao, Qing Yan, Mingyuan Zhuang, Jiejun Chen, Yun Tian, Yingzi Lin, Wei Wu, Hao Li, and Zesheng Dou. 2020. A 12nm programmable convolution-efficient neural-processing-unit chip achieving 825TOPS. In International Solid-State Circuits Conference (ISSCC'20).

[36] Norman P. Jouppi, Doe Hyun Yoon, Matthew Ashcraft, Mark Gottscho, Thomas B. Jablin, George Kurian, James Laudon, Sheng Li, Peter Ma, Xiaoyu Ma, Thomas Norrie, Nishant Patil, Sushma Prasad, Cliff Young, Zongwei Zhou, and David Patterson. 2021. Ten lessons from three generations shaped Google's TPUv4i: Industrial product. In International Symposium on Computer Architecture (ISCA'21). 1-14.

[37] Norman P. Jouppi, Doe Hyun Yoon, George Kurian, Sheng Li, Nishant Patil, James Laudon, Cliff Young, and David Patterson. 2020. A domain-specific supercomputer for training deep neural networks. Communications of the ACM 63, 7 (2020), 67-78.

[38] Norman P. Jouppi, Cliff Young, Nishant Patil, David Patterson, Gaurav Agrawal, Raminder Bajwa, Sarah Bates, Suresh Bhatia, Nan Boden, Al Borchers, Rick Boyle, Pierre-luc Cantin, Clifford Chao, Chris Clark, Jeremy Coriell, Mike Daley, Matt Dau, Jeffrey Dean, Ben Gelb, Tara Vazir Ghaemmaghami, Rajendra Gottipati, William Gulland, Robert Hagmann, C. Richard Ho, Doug Hogberg, John Hu, Robert Hundt, Dan Hurt, Julian Ibarz, Aaron Jaffey, Alek Jaworski, Alexander Kaplan, Harshit Khaitan, Daniel Killebrew, Andy Koch, Naveen Kumar, Steve Lacy, James Laudon, James Law, Diemthu Le, Chris Leary, Zhuyuan Liu, Kyle Lucke, Alan Lundin, Gordon MacKean, Adriana Maggiore, Maire Mahony, Kieran Miller, Rahul Nagarajan, Ravi Narayanaswami, Ray Ni, Kathy Nix, Thomas Norrie, Mark Omernick, Narayana Penukonda, Andy Phelps, Jonathan Ross, Matt Ross, Amir Salek, Emad Samadiani, Chris Severn, Gregory Sizikov, Matthew Snelham, Jed Souter, Dan Steinberg, Andy Swing, Mercedes Tan, Gregory Thorson, Bo Tian, Horia Toma, Erick Tuttle, Vijay Vasudevan, Richard Walter, Walter Wang, Eric Wilcox, and Doe Hyun Yoon. 2017. In-datacenter performance analysis of a tensor processing unit. In International Symposium on Computer Architecture (ISCA'17).

[39] Ajaykumar Kannan, Natalie Enright Jerger, and Gabriel Loh. 2015. Enabling interposer-based disintegration of multicore processors. In International Symposium on Networks-on-Chip (NOCS'15).

[40] Jared Kaplan, Sam McCandlish, Tom Henighan, Tom B. Brown, Benjamin Chess, Rewon Child, Scott Gray, Alec Radford, Jeffrey Wu, and Dario Amodei. 2020. Scaling laws for neural language models. In arXiv preprint arXiv:2001.08361. 
[41] Stephen Keckler, William Dally, Brucek Khailany, Michael Garland, and David Glasco. 2011. GPUs and the future of parallel computing. IEEE Micro 31, 5 (2011), 7-17.

[42] Benjamin Klenk, Nan Jiang, Greg Thorson, and Larry Dennison. 2020. An in-network architecture for accelerating shared-memory multiprocessor collectives. In International Symposium on Computer Architecture (ISCA'20).

[43] Simon Knowles. 2021. Graphcore Colossus Mk2 IPU. In HotChips-33.

[44] Fengfu Li, Bo Zhang, and Bin Liu. 2016. Ternary weight networks. In arXiv:1605.04711.

[45] Youjie Li, Iou-Jen Liu, Yifan Yuan, Deming Chen, Alexander Schwing, and Jian Huang. 2019. Accelerating distributed reinforcement learning with in-switch computing. In International Symposium on Computer Architecture (ISCA'19).

[46] Sean Lie. 2019. Wafer scale deep learning. In HotChips-31.

[47] Sean Lie. 2021. The multi-million core, multi-wafer AI cluster. In HotChips-33.

[48] Mu-Shan Lin, Tze-Chiang Huang, Chien-Chun Tsai, King-Ho Tam, Cheng-Hsiang Hsieh, Tom Chen, Wen-Hung Huang, Jack Hu, Yu-Chi Chen, Sandeep Goel, Chin-Ming Fu, Stefan Rusu, Chao-Chieh Li, Sheng-Yao Yang, Mei Wong, Shu-Chun Yang, and Frank Lee. 2019. A 7nm 4GHz Arm-core-based CoWoS chiplet design for high performance computing. In Symposium on VLSI Circuits.

[49] LLNL. [n.d.]. Laghos. https://computing.llnl.gov/projects/co-design/laghos.

[50] LLNL .2014. The CORAL Benchmarks. https://asc.llnl.gov/CORAL-benchmarks/.

[51] LLNL. 2017. The CORAL2 Benchmarks. https://asc.llnl.gov/coral-2-benchmarks.

[52] Gabriel H. Loh, Natalie Enright Jerger, Ajaykumar Kannan, and Yasuko Eckert. 2015. Interconnect-memory challenges for multi-chip, silicon interposer systems. In International Symposium on Memory Systems.

[53] Peter Mattson, Christine Cheng, Gregory Diamos, Cody Coleman, Paulius Micikevicius, David Patterson, Hanlin Tang, Gu-Yeon Wei, Peter Bailis, Victor Bittorf, David Brooks, Dehao Chen, Debo Dutta, Udit Gupta, Kim Hazelwood, Andy Hock, Xinyuan Huang, Daniel Kang, David Kanter, Naveen Kumar, Jeffery Liao, Deepak Narayanan, Tayo Oguntebi, Gennady Pekhimenko, Lillian Pentecost, Vijay Janapa Reddi, Taylor Robie, Tom St John, Carole-Jean Wu, Lingjie Xu, Cliff Young, and Matei Zaharia. 2020. MLPerf training benchmark. In Machine Learning and Systems (MLSys'20).

[54] Paulius Micikevicius, Sharan Narang, Jonah Alben, Gregory Diamos, Erich Elsen, David Garcia, Boris Ginsburg, Michael Houston, Oleksii Kuchaiev, Ganesh Venkatesh, and Hao Wu. 2018. Mixed precision training. In International Conference on Learning Representations (ICLR'18).

[55] Ugljesa Milic, Oreste Villa, Evgeny Bolotin, Akhil Arunkumar, Eiman Ebrahimi, Aamer Jaleel, Alex Ramirez, and David Nellans. 2017. Beyond the socket: NUMA-aware GPUs. In International Symposium on Microarchitecture (MICRO'17).

[56] MLPerf. 2019. MLPerf Inference Results v0.5. https://mlperf.org/inference-results/.

[57] MLPerf. 2019. MLPerf Training Results v0.6. https://mlperf.org/training-results- $0-6 /$.

[58] Gordon E. Moore. 1965. Cramming more components onto integrated circuits. Electronics 38, 8 (1965), 114-117 (1965).

[59] S. Naffziger, K. Lepak, M. Paraschou, and M. Subramony. 2020. AMD chiplet architecture for high-performance server and desktop products. In International Solid-State Circuits Conference (ISSCC'20).

[60] NASA. [n.d.]. FUN3D. https://fun3d.larc.nasa.gov/.

[61] NVIDIA. 2012. NVIDIA Kepler GK110 Architecture. https://www.nvidia.com/content/PDF/kepler/NVIDIA-KeplerGK110-Architecture-Whitepaper.pdf.

[62] NVIDIA. 2016. NVIDIA NVLink. https://www.nvidia.com/en-us/data-center/nvlink/.

[63] NVIDIA. 2016. NVIDIA Tesla P100 Architecture. https://images.nvidia.com/content/pdf/tesla/whitepaper/pascalarchitecture-whitepaper.pdff.

[64] NVIDIA. 2017. NVIDIA Tesla V100 Architecture. http://images.nvidia.com/content/volta-architecture/pdf/voltaarchitecture-whitepaper.pdf.

[65] NVIDIA. 2019. NVIDIA Turing GPU Architecture. https:/www.nvidia.com/content/dam/en-zz/Solutions/designvisualization/technologies/turing-architecture/NVIDIA-Turing-Architecture-Whitepaper.pdf.

[66] NVIDIA. 2020. NVIDIA A100 Tensor Core GPU Architecture. https://www.nvidia.com/content/dam/en-zz/Solutions/ Data-Center/nvidia-ampere-architecture-whitepaper.pdf.

[67] Open Standards Harmonization Working Group. 2018. Open Specification for a Liquid Cooled Server Rack - Progress Update. https://datacenters.lbl.gov/sites/default/files/OpenSpecification.pdf.

[68] Saptadeep Pal, Eiman Ebrahimi, Arslan Zulfiqar, Yaosheng Fu, Victor Zhang, Szymon Migacz, David Nellans, and Puneet Gupta. 2019. Optimizing Multi-GPU parallelization strategies for deep learning training. IEEE Micro 39, 5 (2019), 91-101.

[69] Vasil Pano, Ragh Kuttappa, and Baris Taskin. 2019. 3D NoCs with Active interposer for multi-die systems. In International Symposium on Networks-on-Chip (NOCS'19).

[70] V. J. Reddi, C. Cheng, D. Kanter, P. Mattson, G. Schmuelling, C. Wu, B. Anderson, M. Breughe, M. Charlebois, W. Chou, R. Chukka, C. Coleman, S. Davis, P. Deng, G. Diamos, J. Duke, D. Fick, J. S. Gardner, I. Hubara, S. Idgunji, T. B. Jablin, J. Jiao, T. S. John, P. Kanwar, D. Lee, J. Liao, A. Lokhmotov, F. Massa, P. Meng, P. Micikevicius, C. Osborne, G. Pekhimenko, A. T. R. Rajan, D. Sequeira, A. Sirasao, F. Sun, H. Tang, M. Thomson, F. Wei, E. Wu, L. Xu, K. Yamada, 
B. Yu, G. Yuan, A. Zhong, P. Zhang, and Y. Zhou. 2020. MLPerf inference benchmark. In International Symposium on Computer Architecture (ISCA'20).

[71] Xiaowei Ren, Daniel Lustig, Evgeny Bolotin, Aamer Jaleel, Oreste Villa, and David Nellans. 2020. HMG: Extending cache coherence protocols across modern hierarchical multi-GPU systems. In International Symposium on HighPerformance Computer Architecture (HPCA'20).

[72] Olaf Ronneberger, Philipp Fischer, and Thomas Brox. 2015. U-Net: convolutional networks for biomedical image segmentation. In arXiv:1505.04597.

[73] Ahmad Sallab, Mohammed Abdou, Etienne Perot, and Senthil Yogamani. 2017. Deep reinforcement learning framework for autonomous driving. Electronic Imaging 19 (2017), 70-76.

[74] Yakun Sophia Shao, Jason Clemons, Rangharajan Venkatesan, Brian Zimmer, Matthew Fojtik, Nan Jiang, Ben Keller, Alicia Klinefelter, Nathaniel Pinckney, Priyanka Raina, Stephen G. Tell, Yanqing Zhang, William J. Dally, Joel Emer, C. Thomas Gray, Brucek Khailany, and Stephen W. Keckler. 2019. Simba: Scaling Deep-Learning Inference with MultiChip-Module-Based Architecture. In International Symposium on Microarchitecture (MICRO'19).

[75] Yi Sun, Yuheng Chen, Xiaogang Wang, and Xiaoou Tang. 2014. Deep learning face representation by joint identification-verification. In International Conference on Neural Information Processing Systems.

[76] Walker J. Turner, John W. Poulton, John M. Wilson, Xi Chen, Stephen G. Tell, Matthew Fojtik, Thomas H. Greer, Brian Zimmer, Sanquan Song, Nikola Nedovic, Sudhir S. Kudva, Sunil R. Sudhakaran, Rizwan Bashirullah, Wenxu Zhao, William J. Dally, and C. Thomas Gray. 2018. Ground-referenced signaling for intra-chip and short-reach chip-to-chip interconnects. In IEEE Custom Integrated Circuits Conference (CICC'18).

[77] V. Vashishtha, M. Vangala, P. Sharma, and L. T. Clark. 2017. Robust 7-nm SRAM design on a predictive PDK. In International Symposium on Circuits and Systems (ISCAS'17).

[78] Oreste Villa, Daniel Lustig, Zi Yan, Evgeny Bolotin, Yaosheng Fu, Niladrish Chatterjee, Nan Jiang, and David Nellans. 2021. Need for speed: Experiences building a trustworthy system-level GPU simulator. In International Symposium on High-Performance Computer Architecture (HPCA'21).

[79] P. Vivet, E. Guthmuller, Y. Thonnart, G. Pillonnet, G. Moritz, I. Miro-Panadès, C. Fuguet, J. Durupt, C. Bernard, D. Varreau, J. Pontes, S. Thuries, D. Coriat, M. Harrand, D. Dutoit, D. Lattard, L. Arnaud, J. Charbonnier, P. Coudrain, A. Garnier, F. Berger, A. Gueugnot, A. Greiner, Q. Meunier, A. Farcy, A. Arriordaz, S. Cheramy, and F. Clermidy. 2020. A 220 GOPS 96 core processor with 6 chiplets 3D-stacked on an active interposer offering $0.6 \mathrm{~ns} / \mathrm{mm}$ latency, $3 \mathrm{~Tb} / \mathrm{s} / \mathrm{mm} 2$ inter-chiplet interconnects and $156 \mathrm{~mW} / \mathrm{mm} 2$ at $82 \%$-peak-efficiency DC converters. In International SolidState Circuits Conference (ISSCC'20).

[80] Tsung Tai Yeh, Matthew D. Sinclair, Bradford M. Beckmann, and Timothy G. Rogers. 2021. Deadline-aware offloading for high-throughput accelerators. In International Symposium on High-Performance Computer Architecture (HPCA'21). 479-492.

[81] Vinson Young, Aamer Jaleel, Evgeny Bolotin, Eiman Ebrahimi, David Nellans, and Oreste Villa. 2018. Combining HW/SW mechanisms to improve NUMA performance of multi-GPU systems. In International Symposium on Microarchitecture (MICRO'18).

Received May 2021; revised August 2021; accepted August 2021 